\title{
Ultrafast Spectroscopy of Photoactive Molecular Systems from First Principles: Where We Stand Today and Where We Are Going
}

\author{
Irene Conti, Giulio Cerullo, Artur Nenov,* and Marco Garavelli* \\ Cite This: J. Am. Chem. Soc. 2020, 142, 16117-16139 \\ Read Online
}

ABSTRACT: Computational spectroscopy is becoming a mandatory tool for the interpretation of the complex, and often congested, spectral maps delivered by modern non-linear multi-pulse techniques. The fields of Electronic Structure Methods, NonAdiabatic Molecular Dynamics, and Theoretical Spectroscopy represent the three pillars of the virtual ultrafast optical spectrometer, able to deliver transient spectra in silico from first principles. A successful simulation strategy requires a synergistic approach that balances between the three fields, each one having its very own challenges and bottlenecks. The aim of this Perspective is to demonstrate that, despite these challenges, an impressive agreement between theory and experiment is achievable now regarding the modeling of ultrafast photoinduced processes in complex molecular architectures. Beyond that, some key recent developments in the three fields are presented that we believe will have major impacts on spectroscopic simulations in the very near future. Potential directions of development, pending challenges, and rising opportunities are illustrated.

\section{INTRODUCTION}

Time-resolved optical spectroscopy is a versatile tool for resolving dynamic processes in molecules both in their ground and excited electronic states. Since the pioneering microsecond flash photolysis experiments, ${ }^{1}$ the generation of ever shorter pulses has furnished researchers with an arsenal of techniques enabling them to access faster and faster timescales, spanning from the nanosecond to the attosecond domains (see, e.g., refs $2 \mathrm{a}$ and $2 \mathrm{~b}$ ). These technological advances have facilitated the study of a broad variety of physical phenomena, from the slowest such as protein conformational changes to the fastest such as electronic dynamics within molecules.

However, it is often not straightforward to interpret the measured time-dependent observables and associate them to specific photophysical/photochemical processes. To address this issue, the progress in experimental techniques has been matched by spectacular advances in $a b$ initio computational methods, which now enable one to study complex molecular systems and to reproduce with nearly quantitative accuracy the measured transient spectra. Theoretical modeling is crucial because it provides the link between the observables and the underlying molecular dynamics, allowing the researcher to interpret the experimental data and to extract from them the maximum amount of information.

This work summarizes some of the most recent developments and results in first-principles modeling of ultrafast spectroscopy of photoinduced processes in complex molecular systems. We show that computational spectroscopy is a mandatory tool for the interpretation of the complex spectral maps delivered by multi-pulse techniques. We consider light pulses spanning different spectral ranges, from $10^{14}-10^{16} \mathrm{~Hz}$ (NIR-Vis-UV), resonant with optical transitions between the valence electronic states of the chromophores up to their photoionization limit, to $10^{17}-10^{18} \mathrm{~Hz}$ (X-rays), which excite core electrons and possess atomic specificity (see Scheme 1). These pulses cover timescales ranging from the attosecond to the picosecond. We discuss experiments performed within the weak-field perturbative regime. Out of the scope of this Perspective are strong electric fields, attosecond electron dynamics, ground-state structural and solvation dynamics, and

Scheme 1. Non-linear Multi-Pulse Techniques

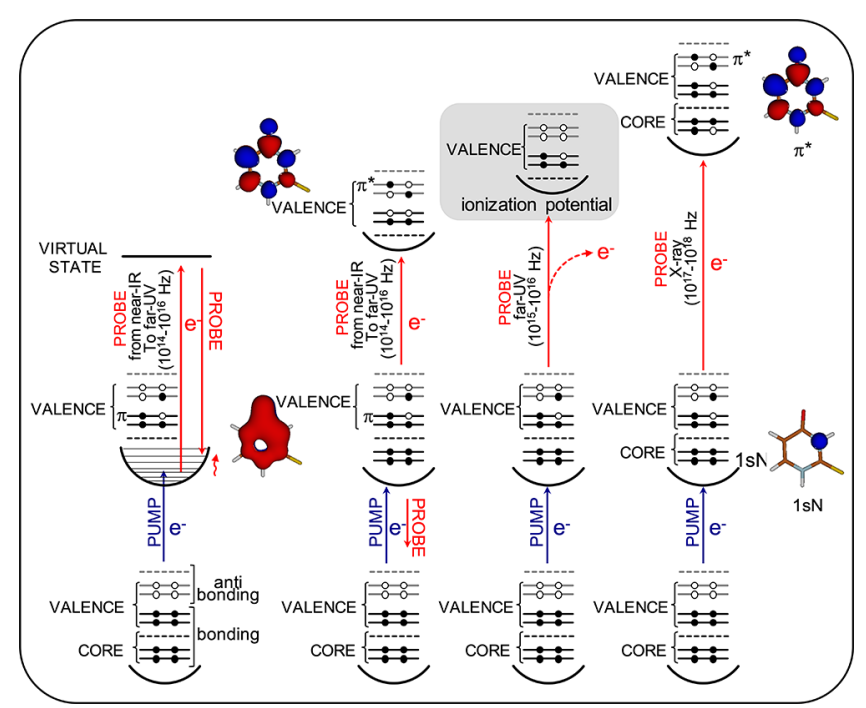

Received: May 6, 2020

Published: August 25, 2020 
Scheme 2. Time-Resolved Multi-Pulse Optical Spectroscopy Simulations: Three Distinct Grand Challenges

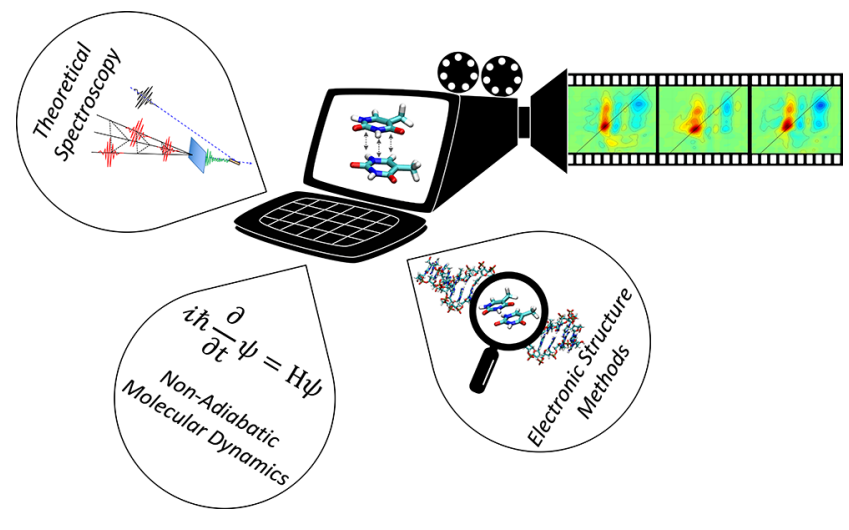

solid-state spectroscopy, while time-resolved vibrational spectroscopy techniques are limited to transient stimulated Raman scattering.

First-principles simulations of time-resolved optical spectroscopy pose three distinct Grand Challenges (see Scheme 2), whose interplay is required to deliver the full picture:

(1) Light-matter interactions need to be modeled. This is the task of Theoretical Spectroscopy, which describes both the perturbation of the electronic density due to the initial photoexcitation (the pump) and the subsequent interaction of the system under study with a delayed light pulse (the probe).

(2) The field-free evolution of the photoexcited system needs to be described by solving the time-dependent Schrödinger equation for the electrons and the nuclei. This is the field of Non-Adiabatic Molecular Dynamics, i.e., propagation of the nuclear wavepacket on the manifold of photochemically relevant electronic states, describing quantum effects like non-adiabatic population transfer, wavepacket splitting, quantum interferences, and tunneling.
(3) Eventually, the potential energy surfaces (PESs) of the electronic states on which the nuclei are moving, and their derivatives and non-adiabatic couplings, need to be obtained by solving the time-independent Schrödinger equation, which is the task of Electronic Structure Methods, whose application range is dictated by the chromophore size.

Each of the three challenges comes with its own problems and bottlenecks: complexity of the spectroscopic experiment to model; incorporation of quantum effects in the dynamics; and breakdown of the adiabatic approximation, duration of the simulations, and trade-off between accuracy and computational cost of the quantum-mechanical (QM) method employed due to the size of the system, just to mention some. Over the years, groundbreaking developments have appeared in each field. This work does not have the ambition to provide a comprehensive review on each of the three Grand Challenges, which would necessarily require distinct and extensive works (see, e.g., ref 3). Rather, it aims at highlighting how the three fields are synergistically combined within an effective computational strategy, a virtual spectrometer, able to deliver experimentally accurate transient spectra in silico. We present key recent advances that we believe will have major impact on spectroscopy simulations. A special focus will be devoted to currently pending conceptual bottlenecks, challenges, and opportunities.

The paper is organized as follows: We first give an overview of the state-of-the-art in the above three fields (see Scheme 2) (section 2); then we show some very recent examples for different types of spectroscopy, covering spectral ranges from the near-IR-Vis-UV to the X-ray, and comparing experiments with theoretical predictions for a variety of photochemical and photophysical problems (sections 3.1 and 3.2). Thereby, we highlight the interplay of the three Grand Challenges in the simulations, as well as the approximations involved and the room left for improvements; we proceed showing theoretical proofs-of-concepts for innovative spectroscopic techniques that demonstrate additional information content (section 3.3); we

\section{Electronic Structure Methods vs System Dimension}

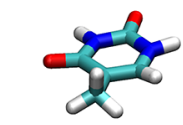

CASSCF/CASPT2 $\mathrm{MRC}$

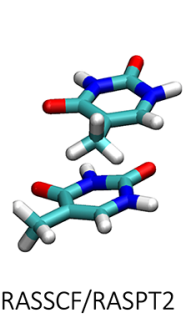

RASSCF/RASPT2

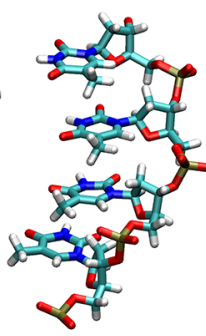

WF in DFT DMRG

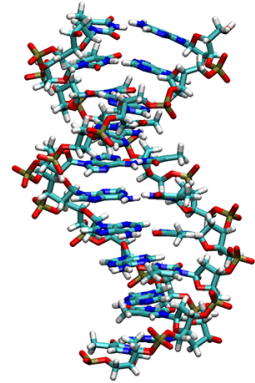

ADC

TDDFT

CC2

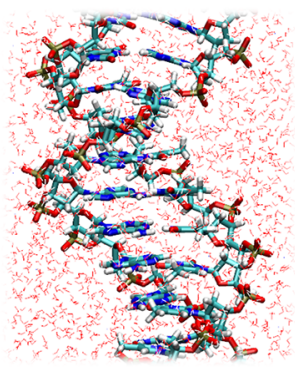

$\mathrm{QM} / \mathrm{MM}$

QM/PCM

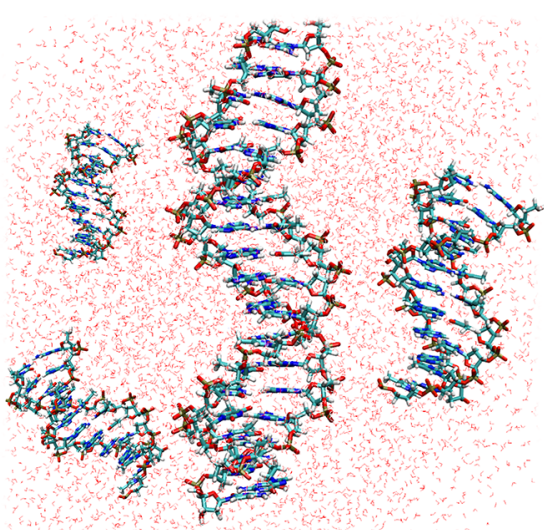

Frenkel exciton Hamiltonian approach

Increasing System Size

Increasing Accuracy of Electronic Structure Method

Figure 1. Range of applicability of Electronic Structure Methods as a function of the system size: DNA as an example. The choice of the computational method is a cost/accuracy trade-off. Details can be found in section 2.1. 
conclude with a critical analysis of the current limitations and future directions of development (section 4).

\section{STATE-OF-THE-ART}

\section{(where the three Grand Challenges meet ... and fight!)}

2.1. The First Grand Challenge: Electronic Structure Methods (that shows that a slimming cure is needed to marry size with accuracy). Recent years have witnessed notable progress in electronic structure computational methods, both wavefunction (WF)-based and density functional theory (DFT)-based, which already today permit the fully quantummechanical computation of system sizes that were unimaginable a decade ago. Concurrently, hardware architecture developments-increasing CPU and RAM capacities, increasing GPU performance, fast input/output rates on solid-state drives, multi-core and multi-node parallelization techniqueshave been exploited to increase speed and efficiency. Notable recent achievements are (i) the implementation of the timedependent (TD) DFT, ${ }^{4 \mathrm{a}} \mathrm{CC}^{4 \mathrm{~b}}$ and $\mathrm{CASSCF}^{4 \mathrm{c}, \mathrm{e}}$ protocols on GPUs, which allows calculations with more than 1000 atoms, ${ }^{4}$ and (ii) the acceleration of the TDDFT ${ }^{4 a}$ and CASSCF ${ }^{5 a}$ / CASPT $2^{5 \mathrm{~b}}$ protocols through massive parallelization, pushing the configuration interaction limit beyond a trillion Slater determinants for CASSCF. ${ }^{5 b}$ By exploiting the steadily increasing performance of supercomputing architectures, codes specifically designed to leverage the advantages of GPUs (e.g., Q-Chem, ${ }^{6 \mathrm{a}}$ Psi4, ${ }^{6 \mathrm{~b}}$ TeraChem $^{6 c}$ ) and massively parallelized supercomputers (e.g., MPQC, ${ }^{6 \mathrm{~d}}$ NWChem, ${ }^{6 \mathrm{e}}$ Octupus $^{4 a}$ ) have appeared.

Beyond profiting from technical hardware innovations, conceptual breakthroughs in WF- and DFT-based theories have been constantly boosting the speed and efficiency, as highlighted in the following subsections correlating specific simulation methods to their appropriate system sizes (see Figure 1).

2.1.1. Multi-Reference Wavefunction-Based Methods. WF-based multi-reference methods provide by construction an unbiased description of the PES of electronic states of arbitrary nature (covalent, ionic, charge-transfer, singly and doubly excited, etc.). Active-space-based approaches, which are the most prominent representatives of this class of methods, suffer from factorial scaling with active-space size, which until recently restrained their application to small and medium-sized molecules. The implementation of density-fitting and Cholesky decomposition techniques ${ }^{7}$ for approximating the electronrepulsion integrals, together with more efficient self-consistent field (SCF) solvers, ${ }^{8}$ makes it possible to handle very large numbers (i.e., thousands) of atomic orbital functions. Novel techniques have emerged recently that solve the configuration interaction problem, one of the major bottlenecks of conventional variational multi-configurational protocols, which currently restricts the active-space size for practical uses to maximally 16 electrons and 16 orbitals. Notable techniques are the density matrix renormalization group (DMRG), which has been applied to CASSCF, ${ }^{9 a}$ CASPT2, 9 b NEVPT2, ${ }^{9 \mathrm{c}}$ and MRCI; ${ }^{9 \mathrm{~d}}$ the full configuration interaction quantum Monte Carlo (FCIQMC) approach combined with CASSCF; ${ }^{9 \mathrm{e}}$ the variational two-electron reduced-densitymatrix-driven (v2RDM)-CASSCF method; ${ }^{9 f}$ the heat-bath configuration interaction (HCI) active-space solver; ${ }^{9 g}$ and the adaptive sampling configuration interaction (ASCI) method. ${ }^{\text {h }}$ These implementations make it possible to handle active spaces with many tens of orbitals. An equally effective approach toward mitigating the factorial increase of the full configuration interaction problem is to remove "configurational deadwood", i.e., configurations which contribute only marginally to the total wavefunction, by introducing a flexible approach to the construction of the active space. Restricted active space (RAS)SCF/RASPT2 and the generalized version GASSCF/GASPT2, ${ }^{10 a}$ as well as the closely related OR$\mathrm{MAS}^{10 \mathrm{~b}} / \mathrm{MRMP}^{10 \mathrm{c}}$ method, also allow construction of active spaces with a few tens of orbitals.

2.1.2. Single-Reference Wavefunction-Based Methods. There are several less costly single-reference WF-based methods for calculating electronic structures, ${ }^{11 a}$ such as the hierarchy of algebraic diagrammatic construction $(\mathrm{ADC})^{11 \mathrm{~b}}$ methods (e.g., $\operatorname{ADC}(2), \operatorname{ADC}(3))$ and the family of coupled cluster (CC $)^{11 c}$ methods (e.g., EOM-CC, LR-CC) that have proven to be reasonably reliable. ${ }^{11 \mathrm{~d}}$ The local variants of these methods, which rely on localized molecular orbitals for the occupied space and pair natural orbitals (PNO), i.e., a compact representation of the virtual space, reduce the scaling of the equations. Recent developments, such as the domain-based local PNO coupled cluster (DLPNO-CC), have reached linear scaling for ground-state problems, ${ }^{11 \mathrm{e}, \mathrm{f}}$ whereas for excited states the efficient computation of PNOs is a matter of intense research. ${ }^{11 \mathrm{~d}}$

While single-reference methods generally provide accurate estimates, they often demonstrate weaknesses in the treatment of some states, e.g., of doubly excited character. Another issue, common to all single-reference methods, is their inability to describe the topology of the PES in the vicinity of the conical intersection (CI) between the excited state and the closed-shell ground state. ${ }^{12}$ The spin-flip family of methods ${ }^{13 a}$ offers a rigorous solution to the problem by starting from a high-spin state to generate the lower-spin ground and excited states by single-electron transitions. Recently, these methods have been systematically applied to SR-CI (SF-CIS ${ }^{13 b}$ ), CC (EOM-SF$\left.\mathrm{CC}^{13 \mathrm{c}}\right)$, and $\mathrm{ADC}\left(\mathrm{SF}-\mathrm{ADC}(3)^{13 \mathrm{~d}}\right)$, although spin-contamination may impair geometry optimization and molecular dynamics. The issue has been resolved through implementation of the spin-complete spin-flip approach (SC-SFORMAS $\left.^{14}\right)$. Multi-reference flavors of the above-mentioned single-reference WF methods (i.e., MR-CC, ${ }^{15 a, b}$ MR-ADC ${ }^{15 c}$ ) have been developed but have yet to mature for routine application. $^{16}$

2.1.3. Density Functional Methods. DFT-based methods for the calculation of excited-state properties combine modest numerical cost and favorable accuracy (since already accounting for correlation energy), thus allowing calculations on larger QM systems as compared to WF-based methods. The linear response TDDFT formulation is nowadays the method of choice for practical applications to excited-state chemistry. However, computational speed comes at the expense of several fundamental shortcomings: (i) transitions with multiple excitations character are not included in TDDFT by definition; (ii) calculations of charge-transfer excitations are particularly challenging due to the inaccurate treatment of long-range correlations; ${ }^{17}$ and (iii) the traditional TDDFT formulation, similarly to other single-reference methods, fails to describe the topology of the PES in the vicinity of the CI between the excited state and the closed-shell ground state. ${ }^{18}$ While a solution to (i) is still out of reach, ${ }^{19}$ long-range corrected functionals ${ }^{20}$ are showing promising (although not conclusive $)^{17}$ results with respect to (ii). ${ }^{21 a, b}$ In particular, we note the Minnesota range-separated hybrid meta functionals ${ }^{21 c}$ 
which provide balanced descriptions of valence, Rydberg, and charge-transfer states for both main-group elements and transition metals. The erroneous CI topology problem (iii) is a sign of the more general difficulty of TDDFT in describing strongly correlated systems and has been addressed by many groups. In the following, we briefly outline several approaches which are ready for routine application by non-experts. One such approach is the spin-flip DFT (SF-DFT) and its spinadapted (spin-complete) version, SA-SF-DFT. ${ }^{22 a-c}$ Another topology-conserving approach is the pseudo-wavefunction (PW) method, which constructs the excited states from a ground-state DFT wavefunction augmented with doubly excited Slater determinants. ${ }^{23 a}$ Finally, a new variant of TDDFT, the dual-functional Tamm-Dancoff approximation (DF-TDA), which employs different functionals for orbital optimization and for Hamiltonian construction, has been shown to recover the correct double cone topology in the vicinity of the $\mathrm{S}_{1} / \mathrm{S}_{0} \mathrm{CI} \mathrm{I}^{23 \mathrm{~b}}$.

2.1.4. BSE/GW Method. The many-body Green's function GW formalism, originally developed for band structure calculations in solid-state physics, ${ }^{24 a, b}$ has attracted the attention of molecular theoretical chemists as an alternative to DFT thanks to its comparable scaling with system size without the need for empirical parameters. Incorporating the Bethe-Salpeter equation (BSE), which describes the bound states of a two-particle system in a relativistic formalism, ${ }^{24 \mathrm{c}}$ the BSE/GW approach can be used to compute the bound states of the photoexcited electron and its hole. ${ }^{24 \mathrm{~b}}$ The BSE/GW approach shows some similarities to TDDFT, like the eigenstate representation in terms of single-electron transitions between occupied and virtual molecular orbitals (thus neglecting transitions with multiple-excitations character) and the scaling with system size. However, the underlying formalism relying on perturbational theory is essentially different with respect to TDDFT, which allows for a more accurate description of charge transfer and Rydberg excitations and a mitigated functional dependence.

2.1.5. WF-in-DFT Embedding Schemes. Substantial efforts have been also recently devoted to combining the advantages of WF and DFT methods. ${ }^{25}$ Ensemble DFT expresses the density of the system as a weighted average of several KohnSham determinants (i.e., an ensemble state), enabling a multiconfigurational DFT treatment. $^{26 a}$ The leading method from this family is the spin-restricted ensemble-referenced KohnSham theory $\left(\right.$ REKS $\left.^{26 \mathrm{~b}}\right)$, which has been extended to include state-interaction along with state-average optimizations (SI-SAREKS). WF-in-DFT embedding schemes tackle the problem of strong interaction by separating the two-electron term in a short-range and a long-range contribution, addressed independently at DFT and WF levels, respectively. ${ }^{27 a}$ A parameter controls the transition between the two regions, making it essentially a semiempirical approach. Such schemes have been reported for CASSCF, CASPT2, QMC, ${ }^{27 b, c}$ DMRG, ${ }^{27 d}$ $\mathrm{CC} 2,{ }^{27 \mathrm{e}}$ and $\mathrm{ADC}{ }^{27 \mathrm{f}}$ Finally, multi-configurational pair DFT (MC-PDFT) extends the original ansatz of Kohn and Sham from a Slater determinant to a multi-configurational wavefunction. ${ }^{28 a}$ Recently, MC-PDFT has been combined with DMRG and v2RDM active-space solvers. ${ }^{28 b, c}$ An excellent review of the state-of-the-art in combining WF and DFT methods can be found in ref 29 .

2.1.6. Hybrid Approaches. Hybrid schemes offer a different perspective to treating large-scale systems, profiting from the localized nature of the electronic excitation. In such cases, a more accurate QM description can be applied to the photoresponsive (reactive) center, while the typically much larger environment, not directly involved in the initial reactive process, is described at a more approximate level. ${ }^{30}$ If the environment is described using molecular mechanics (MM), the method is known as QM/MM. Schemes such as $\mathrm{ONIOM}^{31}$ provide flexible layer compartmentalization, such as $\mathrm{QM}$ (high-level)/QM(low-level)/MM. Conventional QM/ MM implementations work in the so-called "electronic embedding" framework, where the environment is represented as a set of point charges that can polarize the QM core. In recent years, next-generation "polarizable embedding" schemes have been established, relying either on inclusion of atom multi-pole moments in addition to the charges or on polarizable point dipoles, treating the mutual polarization of the QM and MM parts self-consistently. ${ }^{32 a, e}$ Further refinement to the basic "electrostatic embedding" scheme, which resolves the electron "spill-out" problem, has been achieved by the polarizable density embedding method, which introduces an intermediate layer between the $\mathrm{QM}$ and $\mathrm{MM}$ parts represented by its density. ${ }^{33}$

2.1.7. The Frenkel Exciton Hamiltonian. Hybrid schemes offer an appealing way to extend the scale of the calculation at comparably low cost. Yet, when the photoresponsive center is a multi-chromophore aggregate (e.g., light-harvesting complexes, DNA and RNA strands, porphyrin self-assemblies, etc.), excitations can be strongly delocalized over the monomers and/or inter-chromophore charge-transfer states can be photogenerated, thus forcing the QM region to be enlarged. In such cases, first-principles computation becomes intractable. Collective excitations in systems of coupled chromophores can be treated by the so-called Frenkel-Davydov exciton model. ${ }^{34}$ In this formalism, the total wavefunction for the collective system is expanded in the basis of monomer excitations (sites), thus requiring only the (trivially parallelizable) computation of the energies of the individual sites and the inter-site couplings, which are conventionally represented via the Coulomb terms only, thus neglecting exchange couplings due to possible overlap of electronic transition densities of close-lying sites. The progress in size scaling of electronic structure methods has facilitated the benchmarking of exciton models with firstprinciples calculations in small molecular clusters, thus allowing one to study the range of validity of inter-site coupling $^{35}$ approximations and inspiring research toward models beyond Coulomb coupling. A conceptually appealing (but practically ambiguous) way of obtaining electronic couplings lies in the application of diabatization techniques, as the off-diagonal elements of the diabatic Hamiltonian reflect physical quantities such as the probability for energy or electron transfer.

Various diabatization schemes have been proposed over the years: here we note the 4 -fold way, ${ }^{36 a, c}$ the fragment charge difference $^{36 \mathrm{~d}}$ and fragment excitation difference schemes, ${ }^{36 \mathrm{e}}$ Boys localization adapted to many-body problems, ${ }^{36 f, g}$ and the dipole and quadrupole moments (DQ) scheme. ${ }^{36 \mathrm{~h}}$ Herbert and co-workers proposed an $a b$ initio Frenkel-Davydov exciton model (AIFDEM) that avoids, in principle, any approximations $^{22 a}$ by computing explicitly the elements of the full electronic Hamiltonian on the basis of the singly excited Slater determinants. Attempts at incorporating chargetransfer states (not included in the standard Frenkel exciton formulation) have been also recently documented. ${ }^{35 \mathrm{~b}, 37 \mathrm{a}, \mathrm{b}}$ 


\section{Molecular Dynamics simulations}
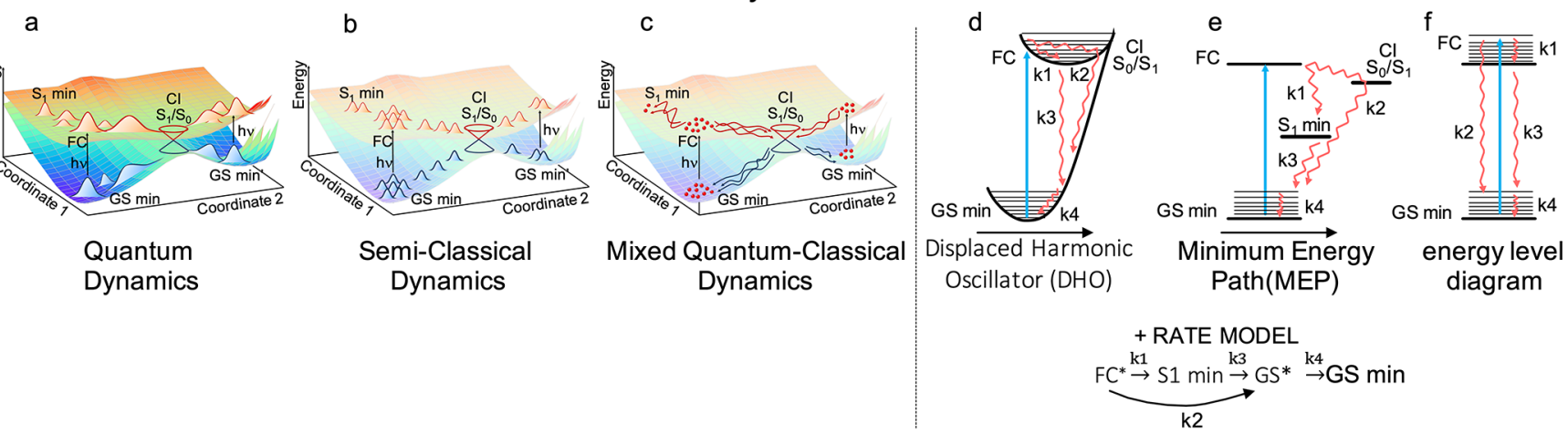

Increasing Accuracy of the Molecular Dynamics Simulation

Increasing Systems Size

Figure 2. Different levels of sophistication in the description of a photoinduced non-adiabatic process: exact quantum dynamics (a), trajectorybased approaches (b, c), model Hamiltonians (d), and static picture based on reaction paths (e) or energy levels diagrams (f). Details on the range of applicability and limitations about the listed methods can be found in section 2.2. GS: ground state; FC: Franck-Condon state. An extended caption for this figure with additional details can be found in the Supporting Information.

2.1.8. Derivatives and Non-adiabatic Couplings. While exploration of the PES topology requires the availability of energy gradients, non-adiabatic couplings (NACs) are essential for the computation of non-adiabatic dynamics. In WF-based methods, analytical gradients and NACs have recently become available at the XMS-CASPT2 ${ }^{38 a, b}$ level, thereby paving the way for simulations of mid-size systems at a dynamically correlated level. Regarding the new generation of active-space solver methods, gradients are available for DMRG-CASSCF ${ }^{38 c}$ and v2RDM-CASSCF. ${ }^{38 \mathrm{~d}}$ In most single-reference methods, gradients are readily available, yet it has been a long-standing issue how to compute NACs. Formulations within the TammDancoff approximation scheme $\mathrm{s}^{36 \mathrm{~g}}$ can be straightforwardly derived for TDDFT, ${ }^{39 a} \mathrm{ADC} 2{ }^{39 \mathrm{~b}}$ and CC2. ${ }^{39 \mathrm{~b}}$ NACs in the framework of EOM-CC were recently reported. ${ }^{39 \mathrm{C}}$ The spinflip version of DFT has also been furnished with NACs, ${ }^{39 d}$ while its spin-adapted upgrade is still lacking. A general formulation of NACs between excited states in TDDFT which avoids the Tamm-Dancoff approximation has recently become possible by means of the PW approach. ${ }^{23 a, 39 e}$ From the plethora of mixed WF/DFT methods, gradients have been reported for subsystem $\mathrm{DFT},{ }^{40 a}$ frozen-density embedding, ${ }^{15 \mathrm{~b}}$ state-specific MC-PDFT, ${ }^{40 \mathrm{~b}}$ and SI-SA-REKS, ${ }^{40 \mathrm{c}}$ although only the latter has been also furnished with NACs. Finally, the lack of analytical gradients for excited states represents a current drawback for the BSE/GW method which prevents its widespread application in the field of photochemistry. ${ }^{40 d}$

2.2. The Second Grand Challenge: Non-adiabatic Molecular Dynamics (to be classical, or not to be classical, this is the question). Molecular dynamics (MD) simulations require solving the time-dependent Schrödinger equation. The Born-Oppenheimer approximation allows one to separate the dynamics of nuclei and electrons, thereby treating the nuclei classically (i.e., applying Newton's equations of motions) and assuming that the electrons equilibrate instantaneously. The Born-Oppenheimer approximation holds for ground-state conformational changes, a typical example of a near-equilibrium dynamics, but breaks down when the system is promoted to an excited state, in which case approximations have to be made elsewhere (see Figure 2).

2.2.1. Quantum Dynamics (QD). The Schrödinger equation can be solved numerically exactly by representing the
Hamiltonian on a grid of time-independent or time-dependent basis functions and propagating their amplitudes, an approach generally labeled as QD (see Figure 2a). The outgoing wave variational principle (OWVP) and R-matrix propagation (RMProp) were originally proposed for treating coupled surfaces in QD. ${ }^{41 a}$ More recent advances in the field led to the time-dependent basis approach, the foundation of the multiconfigurational time-dependent Hartree (MCTDH), ${ }^{41 \mathrm{~b}}$ which enables a more compact description of the nuclear wavepacket with respect to the time-independent basis and, thus, the simultaneous treatment of several tens of degrees of freedom (DOF). ${ }^{41 c, d}$ A multi-layer variant of the $\mathrm{MCTDH}$, where strongly correlated vibrational DOF are contracted, makes it possible to treat systems with several hundreds of vibrational DOF. ${ }^{41 \mathrm{e}}$ Research toward full QD simulation for systems with a large number of DOF has become accessible recently by the time-dependent reformulation of the density matrix renormalization group (TD-DMRG). ${ }^{42}$ Here, only nearest-neighbor interactions are treated explicitly, while the remaining sites are modeled through an effective (i.e., renormalized) basis. This makes TD-DMRG particularly suitable for aggregates dominated by nearest-neighbor interactions. Very recently, surface parametrization methods based on machine learning have been used for improving PESs for QD within a grid-based approach. $^{43}$

Full-dimensional QD based on an analytical representation of the PES within a harmonic approximation has allowed linear absorption simulations with a remarkable accuracy, ${ }^{41 \mathrm{~d}, \mathrm{f,g}}$ while analytical models of the CI topology have given invaluable insights into the non-adiabatic dynamics in its vicinity. ${ }^{41 \mathrm{~h}, 44 \mathrm{a}, \mathrm{b}}$ However, the harmonic picture is often incomplete and inadequate for following the evolution of the system away from the Franck-Condon region toward the photoproducts. In such cases, PESs and NACs are computed for reduced dimensionality (up to three or four degrees of freedom) models, considering only modes essential for describing the process of interest.

One limitation of the grid-based approaches is the difficulty in representing the coupling (dynamical in nature) of the photoactive system to its surrounding; for that reason, QD simulations are typically performed in the gas phase. Recent years have witnessed notable progress in incorporating the 
coupling to the environment, both in a static $45 \mathrm{a}$ and in a dynamic way, ${ }^{45 b, c}$ the latter allowing cases in which solute and solvent dynamics occur on the same timescale to be treated. Another limitation is that the topology of the involved PES must be known in advance, thus calling for "on-the-fly" methods (see below).

2.2.2. Semi-classical Dynamics. Most current developments in the field of MD are focused on protocols which allow the computation of all necessary ingredients for propagating the system "on the fly". One such class of protocols models the nuclear wavepacket evolution by means of frozen Gaussians centered at classical trajectories (see Figure 2b). An example is the variational multi-configurational Gaussian wavepacket (vMCG) ${ }^{46}$ method that is formulated based on MCTDH. The dephasing representation in Gaussian basis (GDR) is conceptually closely related to vMCG, as it also utilizes communicating frozen Gaussian basis functions, yet it is particularly suitable for simulating time-resolved electronic spectra. $^{47 a}$ The method comes with a hierarchy of approximations which bridge the exact with the semi-classical picture. The ab initio multiple spawning (AIMS) ${ }^{3 \mathrm{~d}, 47 \mathrm{~b}}$ methods consider the Gaussian wavepacket evolving through a swarm of independent trajectories, with bifurcations in regions of strong NAC represented through spawning events. The AIMS formulation, which relates to the GDR under well-defined approximations, ${ }^{47 a}$ has itself spawned other approaches, like ab initio multiple cloning (AIMC), which benefits from meanfield (Ehrenfest picture) evolution during periods of strong NAC while simultaneously avoiding mean-field artifacts by flexible expansion of the trajectory basis. ${ }^{48}$

An intriguing recent development concerns the formulation of semi-classical MD simulation protocols within the framework of the exact factorization (EF) formalism. ${ }^{4 a} \mathrm{EF}$ proposes a novel form of the wavefunction to solve the time-dependent Schrödinger equation with the direct consequence that nuclei and electrons are propagated by a set of coupled equations of motion which depend on a time-dependent PES. Promising trajectory-based implementations of EF-the coupled-trajectory mixed quantum-classical (CT-MQC) ${ }^{49 \mathrm{~b}}$ scheme which uses TDDFT as the electronic structure method and the decoherence-induced surface hopping based on exact factorization (DISH-XF) ${ }^{49 c}$ employing CASSCF $/ \mathrm{MRCI}^{49 c}$ or SI-SAREKS $^{49 \mathrm{~d}}$ - demonstrate the capability to capture quantum (de)coherence effects. Reference 3d reviews semi-classical solutions of the time-dependent Schrödinger equation.

2.2.3. Mixed Quantum-Classical Dynamics (MQCD). In the drastic simplification of modeling the evolving wavepacket through a swarm of independent, non-interacting, classical trajectories, one enters the world of mean-field Ehrenfest and trajectory surface hopping (TSH, see Figure 2c). ${ }^{50}$ Owing to its simplicity and remarkably good results, considering the level of approximation adopted, the fewest switches formulation of TSH by Tully (i.e., FSSH) ${ }^{50}$ is nowadays the method of choice for non-adiabatic molecular dynamics simulations, which has been integrated within a vast number of electronic structure methods. ${ }^{3 a}$ Nevertheless, the failure of the prototype formulation to describe decoherence, i.e., the gradual collapse of a coherent quantum-mechanical mixture of electronic states into a classical mixture of pure electronic states, properly (over-coherence problem) $)^{51 \mathrm{a}, \mathrm{c}}$ has spawned in recent years a number of modified protocols. ${ }^{3 \mathrm{a}, \mathrm{b}, 51 \mathrm{~d}, \mathrm{f}}$ Furthermore, considerable attention has been devoted to purely practical issues, common to trajectory-based approaches in general. One such issue stems from the steep shape of the NACs in space and the risk of missing them when employing large time steps in the propagation of the nuclei. Norm-preserving interpolation of the time-dependent overlap, ${ }^{52 a}$ local diabatization, ${ }^{52 b}$ and adaptive time-step protocols ${ }^{52 c}$ have been suggested to remedy the issue. An excellent review on trajectory-based non-adiabatic mixed quantum-classical methods is given in ref $3 \mathrm{a}$.

More recently, the FSSH framework has been extended to model Frenkel exciton dynamics in molecular aggregates for studying charge and excitation energy transfer processes by formulating expressions for gradients and coupling terms. ${ }^{3 e, 53}$

2.2.4. Dynamics Based on Model Hamiltonians. Despite the progress in electronic structure calculations, the cost associated with the calculation of all the necessary ingredients for performing first-principles MD limits the accessible time window of the "on-the-fly" simulations. An alternative ansatz for describing the dynamics of electronically excited states relies on model Hamiltonians which parametrize the coupling to the bath of vibrational degrees of freedom (both intramolecular and with the environment) by frequencydependent spectral densities that indicate the strength of the coupling of a given electronic state to the bath. Thereby, the bath is normally assumed to be harmonic and the coupling linear, an approximation known as the displaced harmonic oscillator (DHO) model, which greatly simplifies the problem, as many quantities of interest can be computed analytically. ${ }^{54}$

In the treatment of multi-chromophoric systems with parametrized Hamiltonians, the system-bath coupling is still handled within the DHO framework. The Hamiltonian contains an additional coupling term, the inter-chromophore coupling. Depending on its strength, the aggregate can be modeled either in a localized (site) basis ("weak" coupling regime) or in a delocalized (exciton) basis ("strong" coupling regime). ${ }^{3 f}$ Different theories describe energy and/or electrontransfer dynamics in these contrasting regimes: as incoherent hops between sites in the Förster model, ${ }^{55 a, b}$ which treats the inter-chromophore couplings perturbatively, and as relaxation between exciton states in the Redfield theory, ${ }^{56,55 b}$ which instead treats the coupling to the bath perturbatively. Modifications of both formulations (e.g., the generalized Foerster $^{55 \mathrm{c}}$ and modified Redfield ${ }^{56 \mathrm{~b}-\mathrm{d}^{\circ}}$ theories) aim at expanding their application range.

A number of methods have been developed that go beyond the above-mentioned perturbative treatments (still within the DHO framework). Methods like the hierarchical equations of motion (HEOM), ${ }^{57 a}$ the iterative quasi-adiabatic propagator path integral (i-QUAPI), ${ }^{57 \mathrm{~b}}$ and the partially linearized density matrix (PLDM) dynamics ${ }^{57 c}$ are considered superior. However, they become computationally much more demanding and therefore limited to specific forms of the spectral density. Implementations to massively parallelized supercomputer architectures, GPU-acceleration, as well as tractable approximations are required for their practical applications. ${ }^{57 \mathrm{~d}-\mathrm{g}}$

2.3. The Third Grand Challenge: Theoretical Spectroscopy (where theory paints the light and matter kiss: with which results, you'll judge!). Most ultrafast optical spectroscopy experiments detect the time-dependent thirdorder non-linear optical response of the system under study. In a general realization, a first actinic light pulse sequence (the "pump") perturbs the electronic density of the system, initiating its dynamics. After a delay, another sequence of pulses (the "probe") is used to create a non-linear polarization in the system emitting a field, the response, which is recorded 


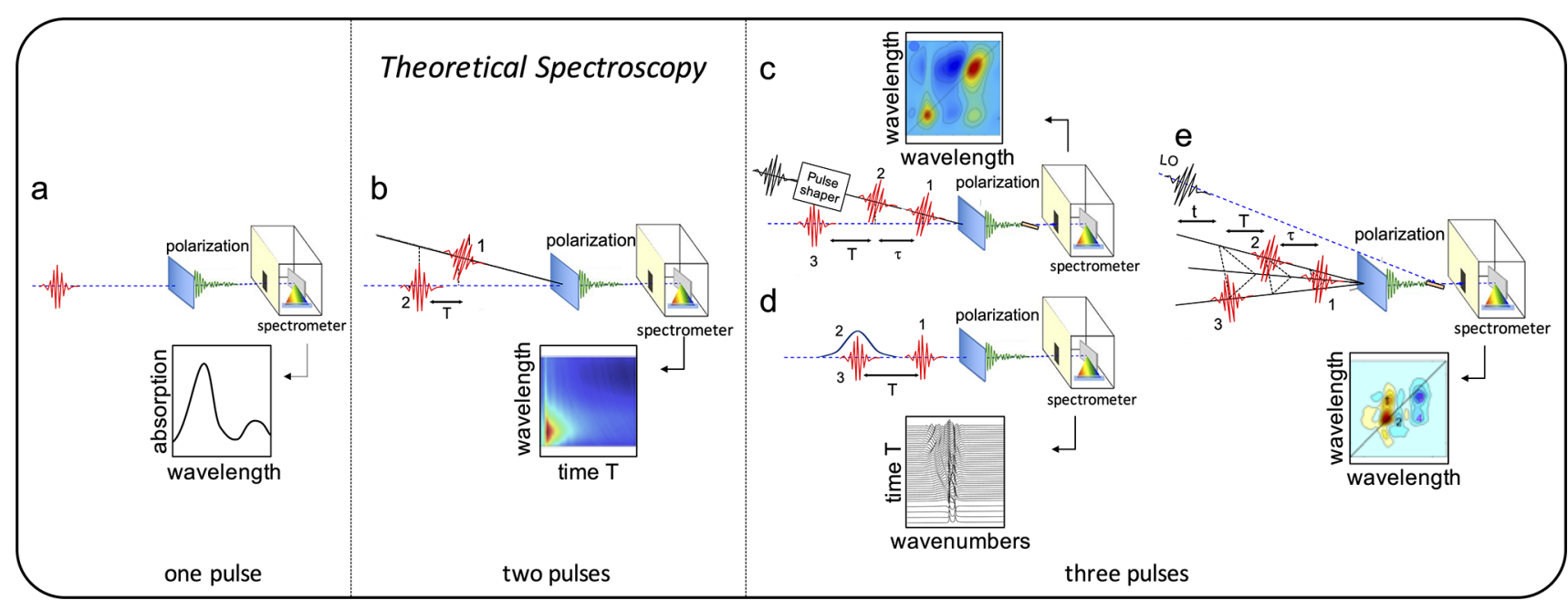

Figure 3. Experimental set-ups for linear and non-linear optical spectroscopy: (a) one-pulse (linear), (b) two-pulse (pump-probe), and (c-e) three-pulse (two-dimensional) spectroscopy. Examples of recent simulations of pump-probe, two-dimensional, and time-resolved Raman spectroscopy are provided in sections 3.1, 3.2 and 3.3, respectively. LO: local oscillator. An extended caption for this figure with additional details can be found in the Supporting Information.

by the detector (see Figure 3). Such light-matter interactions are the object of Theoretical Spectroscopy, whose aim is to identify spectroscopic observables, to elaborate the mathematical framework for their simulation, and to relate the line shapes and evolution of the spectral features to the underlying molecular processes. Theoretical spectroscopy also allows designing tailored pulse sequences aimed at highlighting novel observables, increasing signal-to-noise ratio, and suppressing undesired background signals, profiting from the tunability of pulse parameters (such as duration, central frequency, bandwidth, and polarization) while respecting technical constraints in the preparation of the pulses.

2.3.1. Simulating the System's Response with Quantum Dynamics. In the most general representation, the $n$th order polarization (with respect to the external driving field) induced in the system by the sequence of electric fields can be treated non-perturbatively by incorporating the fields into the system's Hamiltonian. This method permits the exact numerical simulation of the dynamics of the driven system for fields of arbitrary strength and duration. ${ }^{58}$ The non-perturbative approach makes it possible to study, without any approximation, the effects of pulse parameters like central frequency, bandwidth, etc., including strong laser fields and temporally overlapping pulses.

More often the field-matter interaction is treated in the perturbative (i.e., weak field) regime, which is the topic of this Perspective. The $n$th order term of the polarization is expressed as the sum of $n$-dimensional time integrals over non-linear response functions given in terms of quantum-mechanical dipole correlation functions. Within the perturbative treatment, pulses are commonly assumed to be temporally non-overlapping and described by Dirac delta functions, i.e., short compared to the investigated dynamics, which simplifies drastically the calculations. Another advantage of the perturbative treatment is that different response functions can be computed separately, providing a convenient theoretical framework for their classification. ${ }^{54,59,60}$

In principle, the master equations derived from the perturbative and non-perturbative treatment can be solved exactly quantum-mechanically, given the knowledge of the system Hamiltonian. The bottleneck of this fully quantum- mechanical wavepacket propagation is the high cost of precomputing the PES, which limits its use to simple models, such as di- and triatomics, as well as some elementary chromophores (ethylene, formaldehyde, pyrazine).41d,61

2.3.2. Simulating the System's Response with TrajectoryBased Approaches. A more convenient approach for solving the equations involves the semi-classical stochastic modeling of bath fluctuations. Thereby, the response function is calculated by averaging over an ensemble of realizations, propagated through the trajectory-based MD approaches described in the previous section (sub-sections 2.2.2 and 2.2.3). Apart from the considerable computational cost associated with the propagation of a swarm of trajectories, semi-classical methods must confront the issue of defining the density matrix and treating the quantum feedback on the classical bath during a coherence state evolution, a problem particularly severe in surface hopping formulations. ${ }^{51 b, 62}$ Trajectory-based implementations of linear absorption, ${ }^{63}$ transient absorption and emission, photoionization spectroscopy, ${ }^{47 a, 63 b, c}$ stimulated Raman, ${ }^{63 d-f}$ and two-dimensional electronic spectroscopy (2DES) $)^{63 g-i}$ that address these issues to various levels of sophistication have been documented. Due to the high cost associated with the more accurate description of coherences, most simulations adopt the semi-classical Franck-Condon (also called "snapshot") approximation, neglecting the time-dependence of the observable of interest during the coherence evolution, an approximation strictly valid only when the value of the observable fluctuates slowly with respect to nuclear relaxation. ${ }^{63 \mathrm{~g}, 64 \mathrm{a}, \mathrm{b}}$ Due to its limitations, the snapshot approximation is more useful for resolving spectral features of ground-state conformational dynamics $^{64 c, d}$ and long-living excited states. $^{64 \mathrm{~d}, \mathrm{e}}$

2.3.3. Simulating the System's Response with Model Hamiltonians. The linear and non-linear optical responses can be modeled analytically for Hamiltonians constructed within the harmonic approximation (DHO model, see section 2.2.4) relying on the cumulant expansion of Gaussian fluctuations (CGF) to second order. ${ }^{54}$ The spectrum is computed as a sum over all states (SOS), each one characterized by its energy, oscillator strength, and spectral density, which give rise to the signal's position, intensity, and line shape, respectively (see 


\section{Two-dimensional Electronic Spectroscopy (2DES) Simulations}
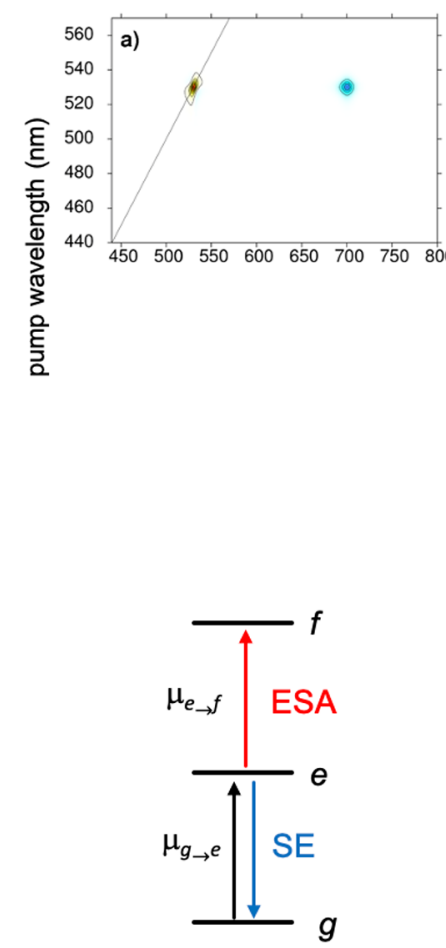

Electronic structure + TDM
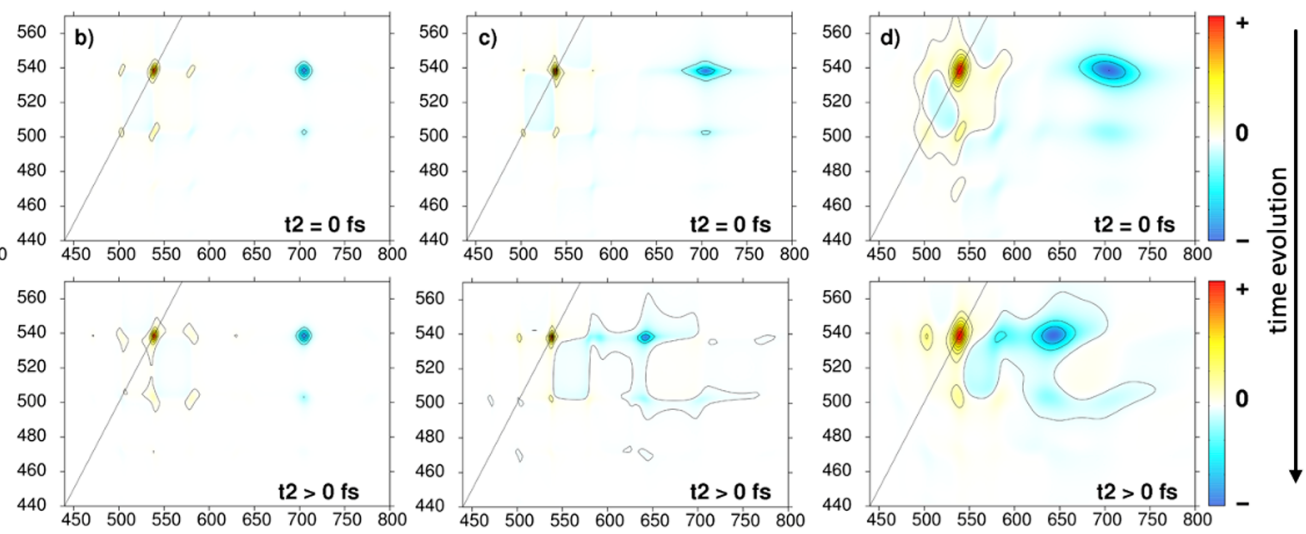

probe wavelength $(\mathrm{nm})$

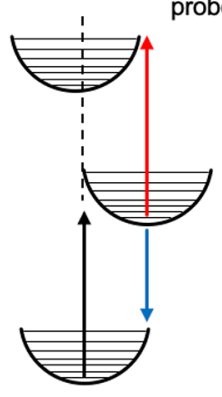

Vibrational states

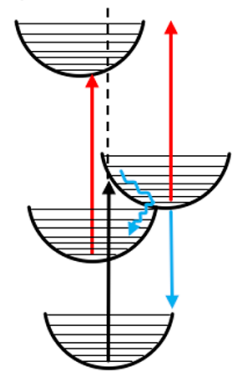

Population dynamics + state specific signatures

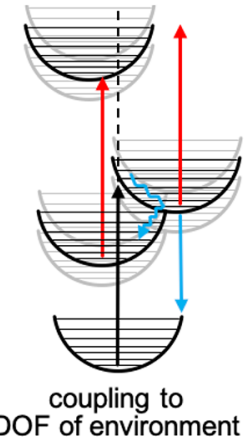

DOF of environment

increasing complexity of the simulation

Figure 4. Various flavors of increasing accuracy in the simulation of two-dimensional electronic spectroscopy (2DES): (a) stick spectrum, (b) plus coupling of the electronic and intramolecular vibrational degrees of freedom, (c) plus electronic population dynamics, (d) plus coupling of the continuum of degrees of freedom of the environment. SE: stimulated emission; ESA: excited-state absorption. An extended caption for this figure with additional details can be found in the Supporting Information.

Figure 4 for a demonstration of the influence of the different approximations on the simulation of the signal line shapes). The CGF formulation is exact for adiabatic dynamics, while non-adiabatic effects can be included at various levels of sophistication. ${ }^{65 a}$ Generalizations beyond the harmonic approximation $^{65 \mathrm{~b}}$ and weak system-bath coupling have also been reported recently. ${ }^{65 c}$

For strongly coupled aggregates (Frenkel-Davydov exciton model, see section 2.1.7), the SOS approach quickly becomes too expensive as the number of excited states grows quadratically with the system size. To circumvent this, a quasi-particle formulation of the CGF method has been developed which scales more favorably with system size. ${ }^{60,66}$ Yet, it exhibits some limitations considering the treatment of non-adiabatic effects.

Until recently, the practical application of the above theories relied on empirical parameters obtained experimentally or on the basis of simplified molecular models. The past few years have witnessed a shift toward a fully computational estimate of the required ingredients: couplings to discrete intramolecular vibrational degrees of freedom via projection methods, ${ }^{67 a-c}$ couplings to the continuum of solvent vibrational degrees of freedom from MD simulations, ${ }^{34,57 \mathrm{c}}$ and electronic structure of fundamental chromophores with state-of-the-art QM methods. $^{3 \mathrm{i}}$

2.3.4. Modeling Spectral Signatures. Besides the level of precision of the underlying $\mathrm{MD}$, another bottleneck in the simulation of time-resolved electronic spectroscopy is the cost associated with the computation of the measured physical quantities: normal-mode frequencies, excited-state absorptions and transition dipole moments in the visible and ultraviolet, ionic-state energies in photoelectron spectroscopy, corevalence transitions in resonant inelastic X-ray scattering, transition densities in X-ray diffraction, and electronic polarizabilities in attosecond Raman spectroscopy. Recent years have witnessed tremendous progress in the development of protocols for monitoring these quantities along the dynamics simulation. Protocols for computing the optical response of excited states in conjugated polyenes and aromatic compounds were developed within the framework of multiconfigurational wavefunction theory. ${ }^{68}$ The multi-configurational nature of excited states is notoriously a problem for DFT. A recent method for computing excited-state absorptions combines real-time (RT) and linear-response (LR) TDDFT. ${ }^{69}$ Another approach computes Dyson norms between neutral and ionic states within multi-configurational wavefunction theory, as required for obtaining signal intensities in photoelectron spectroscopy ${ }^{70}$ (section 3.1). A simple but elegant projection technique, in combination with RASSCF theory, has made accessible single and multiple core-valence transition energies and dipole moments. ${ }^{71}$

\section{APPLICATIONS}

(where, after fighting, the three Grand Challenges cooperate) 


\section{Applications and Perspectives Distribution in the Grand Challenges Space}

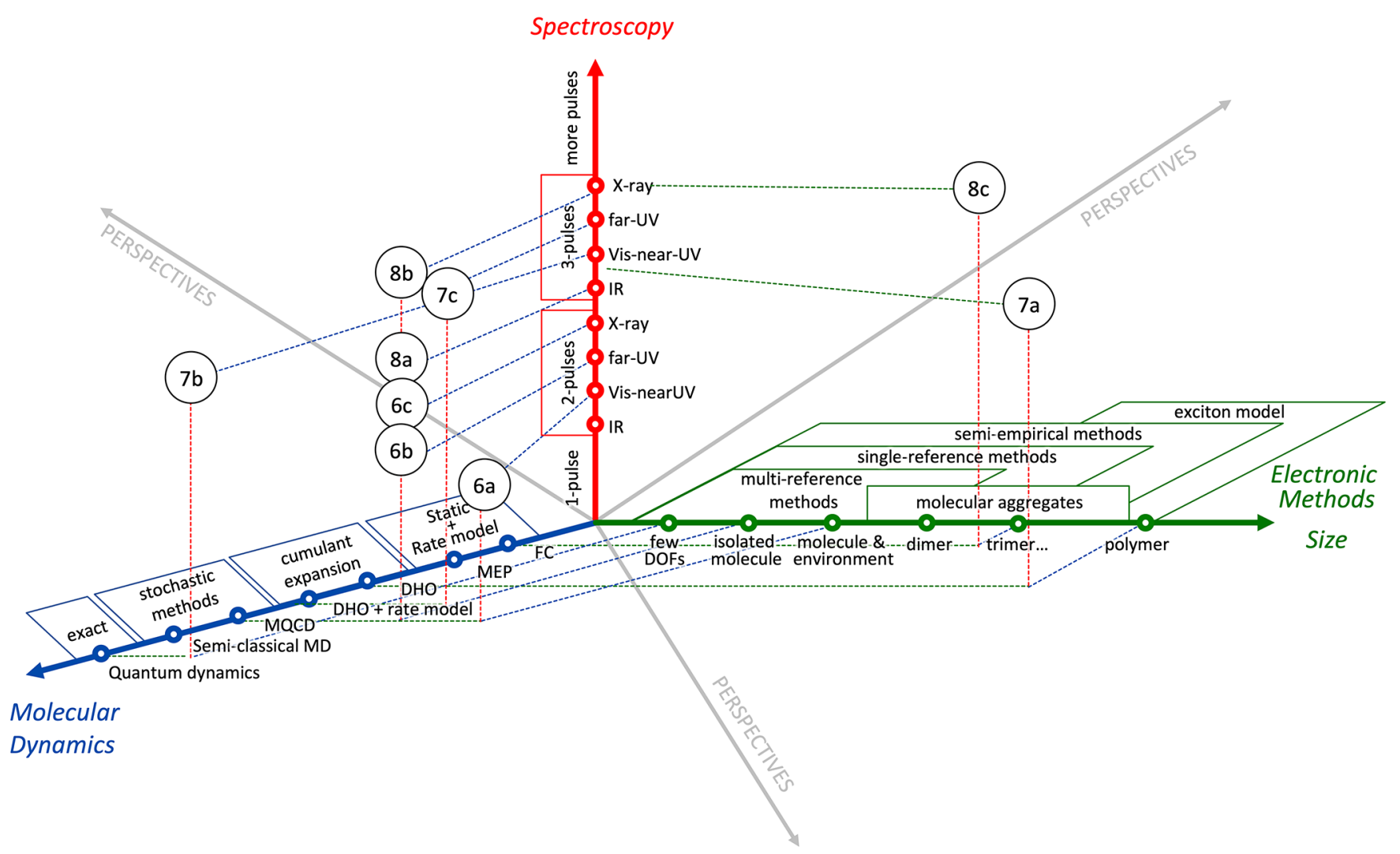

Figure 5. Schematic representation of the three Grand Challenges: choosing a suitable electronic structure method to handle the dimensionality of the system (green axis), describing the non-adiabatic molecular dynamics (blue arrow), and implementing protocols for various types of non-linear spectroscopy (red arrow). Circles with labels refer to examples discussed in section 3 and shown in Figures 6, 7, and 8.

In the previous section, we reviewed the most recent developments in the fields of electronic structure theory, molecular dynamics simulations, and theoretical spectroscopy. In this section, we demonstrate, based on selected examples, how these fields are forced to cooperate to produce from first principles time-dependent molecular spectra which can be directly compared to the experimental results. In the first two subsections, we show a one-to-one comparison between theory and experiment for non-linear two-pulse (i.e., pump-probe, section 3.1) and three-pulse (i.e., two-dimensional, section 3.2) spectroscopy (see Figure 3). The reported examples set the current state-of-the-art and the frontier between "explored" and "unexplored" territories, as shown in Figure 5. Therefore, when discussing these cases, ongoing challenges and directions for future improvements are highlighted. In section 3.3, we demonstrate the capabilities of theory to envision novel spectroscopic techniques, whose benefits will likely trigger future experiments.

3.1. Pump-Probe Spectroscopy. Pump-probe spectroscopy (see Figure $3 \mathrm{~b}$ ), also known as transient absorption (TA), is a widespread technique for studying MD thanks to its straightforward experimental realization and the ease to interpret the spectra. Over the years, simulations have evolved to a level where TA spectra of small and medium-size chromophores (up to 20-30 atoms) can already be computed very accurately from first principles, thereby revealing the physical phenomena behind the spectroscopic signatures.
Below, the state-of-the-art is illustrated by three very recent examples.

3.1.1. UV Pump, Vis-UV Probe. Broadband (Vis-UV) TA spectroscopy simulations were combined with hybrid QM/ MM mixed quantum-classical trajectory-based non-adiabatic dynamics to study the ultrafast excited-state dynamics of the sulfur substituted nucleobase 4-thiouracil in water (Figure 6a). ${ }^{67 a, 72}$ Electronic structure calculations were done at the multi-configurational second order perturbation (CASPT2) level of theory. The results explain the reason for the experimental mismatch between the timescales for the decay of the stimulated emission (SE) from the bright $\pi \pi^{*}$ state (76 fs) and the rise of the excited-state absorption (ESA) of the triplet state (225 fs). The work provides compelling evidence that the intersystem crossing occurs via an intermediate dark state of $n \pi^{*}$ nature previously reported in gas-phase studies. ${ }^{73}$ Transient spectra within the CGF method parametrized on the basis of the simulated dynamics reproduce all characteristic spectral features, such as the origin of the ESA and the intensity beats in the SE.

3.1.2. Time-Resolved Photoelectron Spectroscopy in the Far-UV. Time-resolved photoelectron spectroscopy (TRPES) simulations were performed on the keto and enol tautomers of cytosine (Figure 6b). ${ }^{74}$ Theoretical spectra adopting the snapshot approximation were obtained by computing the Dyson norms between the singlet/triplet states of the neutral and the doublet/quartet states of the ionized species along gasphase trajectory-based dynamics on the neutral species within 
PUMP-PROBE SPECTROSCOPY

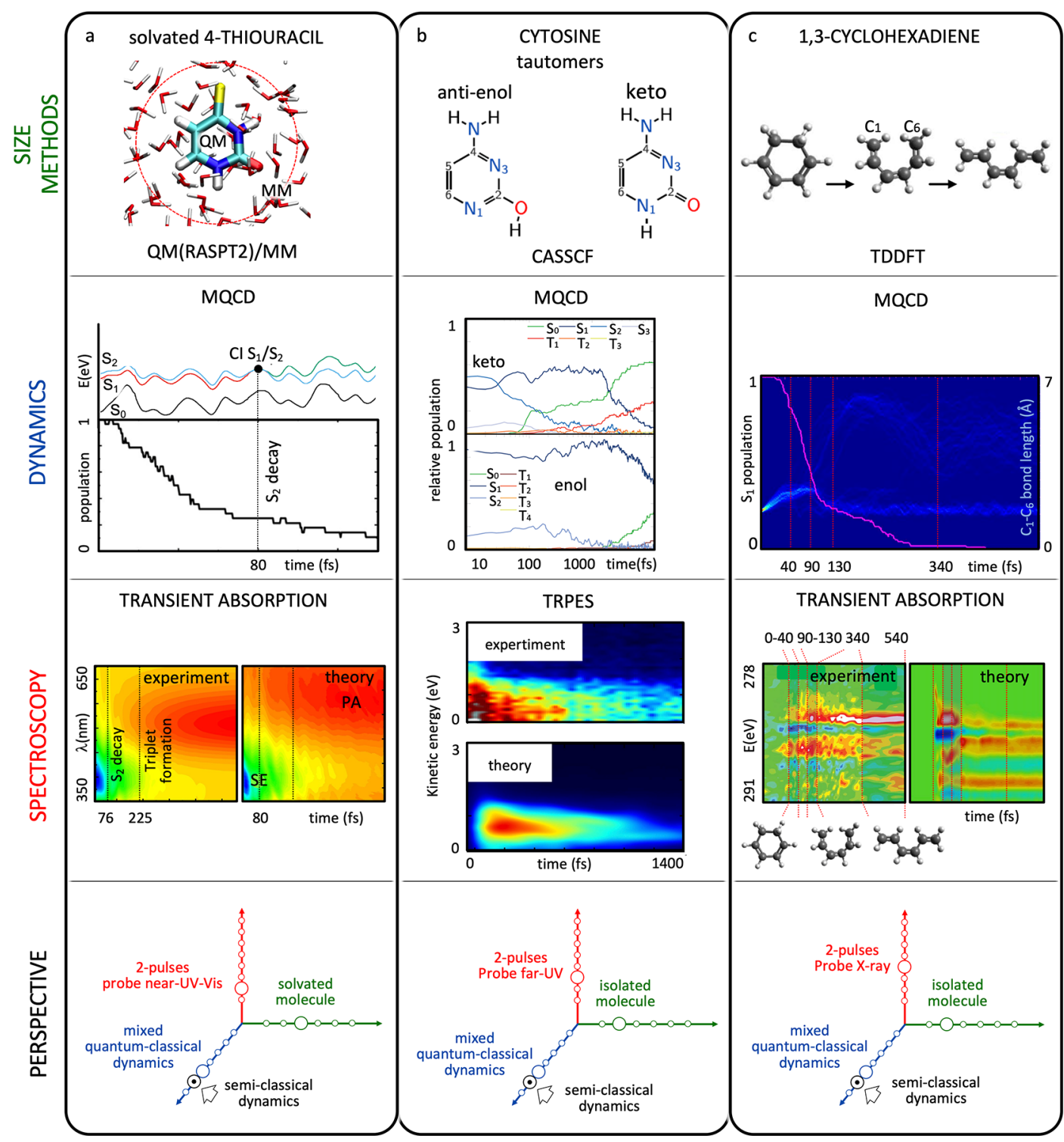

Figure 6. Three examples of pump-probe spectroscopy simulations from first principles and comparison to experiments: (a) solvated thiouracil (ref 67a), (b) isolated cytosine (ref 74), and (c) isolated cyclohexadiene (ref 76). The state-of-the-art of the simulation in terms of system size/ electronic structure method, molecular dynamics protocol, and spectroscopy technique are shown in the schematic representation of the three Grand Challenges (see Figure 5). Advancements in the simulations that would improve the agreement with the experiment are indicated with a black arrow. An extended caption for this figure with additional details can be found in the Supporting Information. Figure rights: (a) Adapted with permission from ref 67a. Copyright 2018 American Chemical Society. (b) Adapted with permission from ref 74a. Licensed under CC BY 4.0. Copyright 2016 Springer Nature. Reprinted in part with permission from ref 74b. Copyright 2013 Wiley-VCH Verlag GmbH \& Co. KGaA, Weinheim. (c) Adapted with permission from ref 76. Copyright 2017 American Association for the Advancement of Science.

TSSH framework, explicitly considering intersystem crossing events. Electronic structure calculations were carried out at CASSCF level of theory. The photoexcited bright $\pi \pi^{*}$ state initially relaxes to the singlet $\mathrm{n} \pi^{*}$ state, from which it bifurcates into channels which either explore the CI seam with the ground state or lead to the population of a triplet state. The two tautomers are found to decay through different regions of the seam. These competing processes occur on similar timescales, so that the different relaxation pathways are completely obscured in the total photoionization signal. This calls for the design of new experimental setups capable of discriminating the individual channels.

3.1.3. UV Pump, X-ray Probe. The light-activated electrocyclic ring-opening process of 1,3-cyclohexadiene represents a prototypical photochemical pericyclic reaction mediated by a $\mathrm{CI}^{75}$ Soft X-ray spectroscopy simulations on top of nonadiabatic mixed quantum-classical molecular dynamics were able to reproduce the experimentally observed spectroscopic signature near the carbon K-edge $(\sim 284 \mathrm{eV})$, a fingerprint of the population of the intermediate pericyclic minimum (Figure 6c). ${ }^{76}$ TDDFT was employed to simulate core-valence transitions along the trajectories. The simulations reproduced the timescale of the electronic rearrangement required to initiate the pericyclic reaction $(\sim 60 \mathrm{fs})$, as well as the lifetime of the excited state $(\sim 110 \mathrm{fs})$.

The above-mentioned examples, covering a broad spectral interval from the visible to the soft X-ray, show a striking agreement between theory and simulation. Such agreement is 
TWO-DIMENSIONAL SPECTROSCOPY

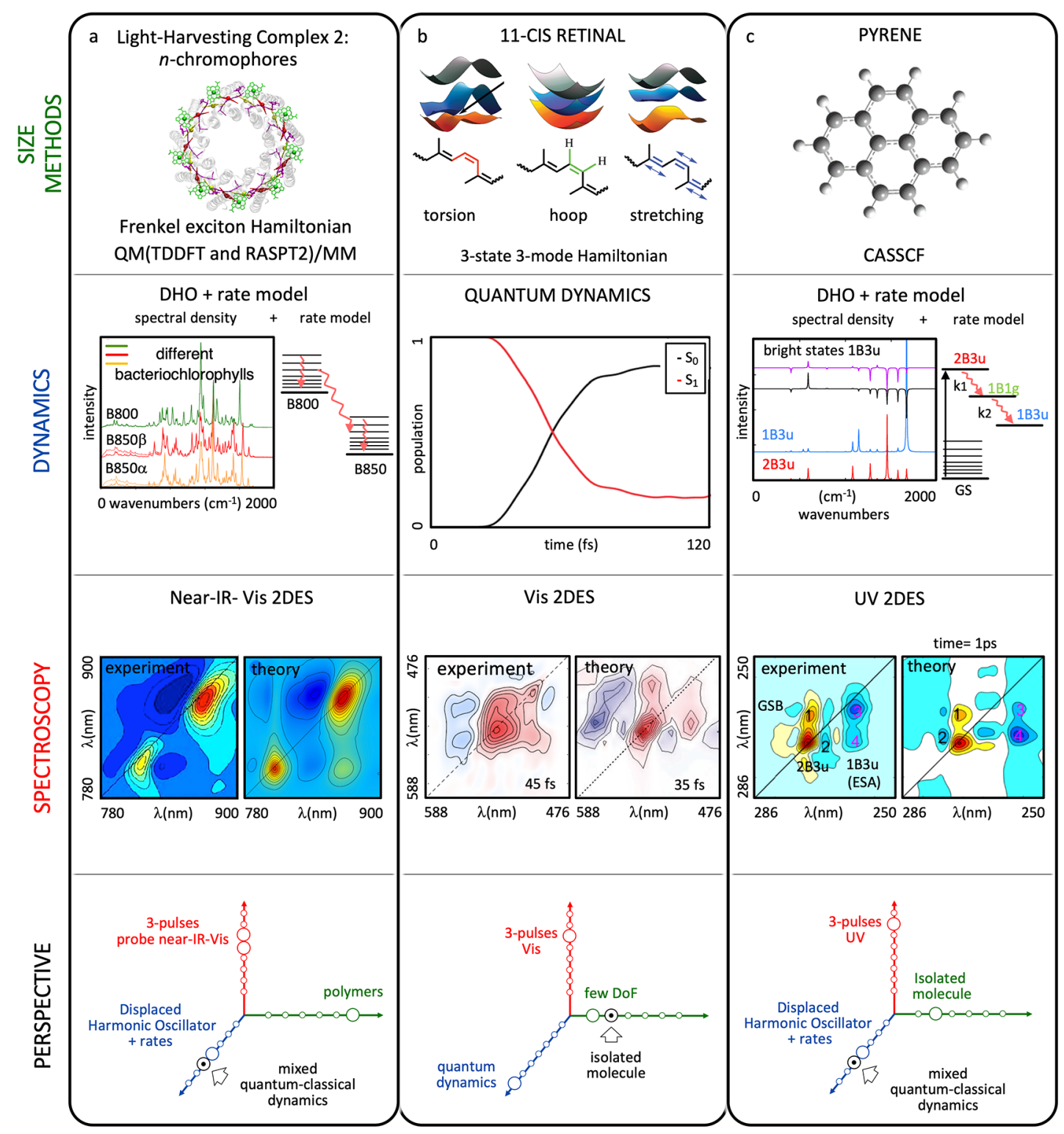

Figure 7. Three examples of two-dimensional electronic spectroscopy (2DES) simulations from first principles and comparison to experiments: (a) light-harvesting complex 2 (ref 79), (b) retinal protonated Schiff base (refs 81 and 82), and (c) pyrene (ref 67c). The state-of-the-art of the simulation in terms of system size/electronic structure method, molecular dynamics protocol, and spectroscopy technique are shown in the schematic representation of the three Grand Challenges (see Figure 5). Advancements in the simulations that would improve the agreement with the experiment are indicated with a black arrow. An extended caption for this figure with additional details can be found in the Supporting Information. Figure rights: (a) Adapted with permission from ref 79. Copyright 2017 American Chemical Society. (b) Adapted by permission of the Royal Society of Chemistry from ref 81 . Experimental spectrum adapted with permission from ref 82. Copyright 2017 American Chemical Society. (c) Adapted with permission from ref $67 \mathrm{c}$.

remarkable in light of the numerous involved approximations. For the future we expect applications to implement advanced semi-classical dynamics simulation protocols beyond the Tully approximation (such as AIMS $^{3 \mathrm{~d}}$ or the emerging schemes based on the exact factorization $\left.(\mathrm{EF})^{49 a}\right)$. A rigorous comparison between various approximations of the nonadiabatic dynamics would allow a better understanding of the range of their validity. Furthermore, we expect computational software to implement the many new tools for easily simulating various TA signal features, thus rendering spectroscopy simulations routinely accessible also to non-experts. This would allow researchers to predict spectral fingerprints of the photoinduced processes and to obtain in silico the optimal pulse parameters (such as wavelength, duration, polarization, etc.) required for their detection in real experiments.

3.2. Two-Dimensional Spectroscopy. Two-dimensional (2D) spectroscopy employs a sequence of properly timed and phase-locked pulses to fully resolve the field emitted by the third-order non-linear polarization of the system (see Figure $3 \mathrm{c}, \mathrm{e})$. Using a Fourier transform approach, 2D spectroscopy makes it possible to correlate spectral signatures to the pump and probe frequencies in a $2 \mathrm{D}$ plot, thus increasing the spectral resolution without sacrificing temporal resolution. 2D spectroscopy is particularly appealing for the study of multichromophoric systems with highly congested spectra which are difficult to interpret with the conventional TA techniques. 
With respect to its one-dimensional counterpart, 2D spectroscopy makes it possible to (a) identify chromophore-specific signatures and inter-chromophore couplings, (b) monitor energy and charge transfer processes between coupled states/ chromophores, and (c) measure separately homogeneous and inhomogeneous contribution to the linewidth of optical transitions and follow their temporal evolution. 2D spectroscopy is nowadays established in the infrared (two-dimensional infrared spectroscopy, 2DIR) and in the visible (2D twodimensional electronic spectroscopy, 2DES) with applications in solvation dynamics, conformational analysis, protein folding and unfolding, and energy and charge transfer. ${ }^{77}$ The required interferometric stability between pulses makes the practical realization of $2 \mathrm{DES}$ techniques at shorter wavelengths (UV and beyond) considerably more difficult. ${ }^{3 \mathrm{~h}}$ The interpretation of the $2 \mathrm{D}$ spectra brings challenges of its own, like the assignment of spectral beats to either electronic coherences or molecular vibrations. ${ }^{78-\mathrm{a}}$ Thus, much of the theoretical effort has been focused on understanding the origin of the signals and the line shapes on simple models. Overall, due to the intricacy of realization and interpretation, only a few works have reported a face-to-face comparison between experiment and simulation. Below, the state-of-the-art is illustrated by three recent examples:

3.2.1. Near-IR-Vis 2DES. Simulations of broadband (nearIR-Vis) 2DES on the light-harvesting complex 2 (LH2) of a purple bacterium integrated quantum chemistry and an electron-phonon exciton model (see Figure 7a). ${ }^{79}$ Electronic structure calculations were executed at both the single(TDDFT) and multi-reference (CASPT2) levels in combination with state-of-the-art models for treating the environment (atomistic and polarizable embeddings). Bath dynamics and exciton transport between the pigments were described by Redfield (strong coupling regime) and Förster (weak coupling regime) theories. The work provides an interpretation of the main features of the 2 DES spectrum at very short times. ${ }^{80}$ In particular, the characteristic energy splitting of the bacteriochlorophyll bands and the vibrational progression in the carotenoid region reinforced the hypothesis of the involvement of a dark intermediate state of the carotenoid in the energytransfer process.

3.2.2. Vis $2 D E S$. Theoretical $2 \mathrm{DES}$ was used to study the photoisomerization of the 11-cis isomer of the retinal chromophore inside the protein rhodopsin (see Figure $7 \mathrm{~b}$ ). ${ }^{81}$ Thereby, the researchers relied on a parametrized three-mode three-state model Hamiltonian which explicitly considers the hydrogen out-of-plane (HOOP) mode besides the torsional and ethylene stretch coordinates, as well as an additional excited state giving rise to ESA in the targeted spectral window. 2DES simulations adopting the perturbative regime on top of exact time-dependent wavepacket propagation conducted with the model Hamiltonian agree reasonably well with experiment and demonstrate that the origin of the observed absorptive spectral features is not the HOOP mode as was previously suggested. ${ }^{81}$ Instead, it was found that an ESA to a higher lying state which is not involved in the isomerization "contaminates" the spectra at early times.

3.2.3. UV 2DES. $2 \mathrm{DES}$ simulations mapped the spectral signatures of the ultrafast excited-state deactivation of pyrene, promoted in its second bright state absorbing in the UV (see Figure $7 \mathrm{c}$ ). ${ }^{67 \mathrm{c}}$ The simulations were conducted with the CGF parametrized by electronic structure calculations at the multiconfigurational second-order perturbation (CASPT2) level.
The simulations resolve the nature of the high-frequency vibrations which give rise to a checkerboard pattern in the experimental spectra, ${ }^{67 \mathrm{c}}$ assigning them to carbon-carbon stretchings. Furthermore, a low-frequency quantum beat arising from an excited-state wavepacket ${ }^{83}$ was matched to an in-plane bending vibration activated upon crossing a CI. The agreement with the experiments ${ }^{3 h}$ demonstrates that wavefunction-based techniques are capable of accurately reproducing the high-energy manifold of organic chromophores.

The presented 2DES simulations show a good agreement with the experimental data, but they have not yet matured to the level of TA simulations. The spectra lack occasionally relevant features (e.g., ESA peaks), and the modeling of the signal line shape is less satisfactory owing to the drastic approximations unavoidable to tackle the increased system complexity. For the future we expect applications to consider in a rigorous way transitions to higher lying states, giving rise to significant ESA contributions. ${ }^{67 c, 79,81}$ Furthermore, we expect applications to employ advanced dynamics simulation protocols to describe exciton energy and charge transport, as well as relaxation pathways that go beyond the Redfield and CGF approximations. This undertaking seems more and more feasible with the recent extension of the FSSH framework to exciton dynamics ${ }^{3 e, 53}$ and trajectory-based 2DES simulation protocols. $^{63 \mathrm{~g}-\mathrm{i}}$

3.3. Novel Techniques. With the advances in computational technologies and the developments in theoretical and experimental ultrafast spectroscopy, the synergy between experiment and theory is steadily increasingly. On the one side, simulations are not plagued by the technical limitations accompanying the experiments (e.g., limited pulse bandwidth, low phase stability, low signal-to-noise ratio, solvent artifacts, etc.). Complex pulse sequences can be conceived to resolve fingerprints of the process under investigation, thus inspiring experimentalists to search for ways to overcome the restrictions. For instance, three-pulse femtosecond and attosecond stimulated Raman spectroscopy techniques have been recently proposed. Very remarkably, off-resonant attosecond stimulated Raman spectroscopy, also known as transient redistribution of ultrafast electronic coherences in attosecond Raman signals (TRUECARS), holds the promise to have the necessary temporal resolution and the sensitivity to resolve the passage of a wavepacket through a $\mathrm{CI}^{84}$ On the other hand, experimental breakthroughs (e.g., the X-ray free electron laser) allow scientists to explore previously inaccessible spectral regions and to resolve novel phenomena, thus stimulating theoretical efforts in rationalizing the observations and in conceiving novel techniques that exploit the new laser sources. For instance, recent advances in X-ray laser technology reporting bright and ultrashort pulses ${ }^{85 a-c}$ have sprung a number of non-linear techniques exploiting the broad bandwidth, short duration, spatial localization of the excitation, and element specificity of exahertz pulses. ${ }^{3 g, j}$ Among these techniques we note (a) X-ray TA spectroscopy, the counterpart of optical TA spectroscopy, ${ }^{86 a-c}$ which has already been successfully implemented experimentally; ${ }^{76,87}$ (b) $2 \mathrm{D}$ coherent $\mathrm{X}$-ray spectroscopy, ${ }^{86 \mathrm{~d}-\mathrm{f}}$ which extends the benefits of $2 \mathrm{DES}$ to this frequency range; and (c) time-resolved X-ray diffraction, which allows us to resolve MD in time and space. ${ }^{86-j}$

In the following, we present three examples which illustrate some of these novel techniques. 


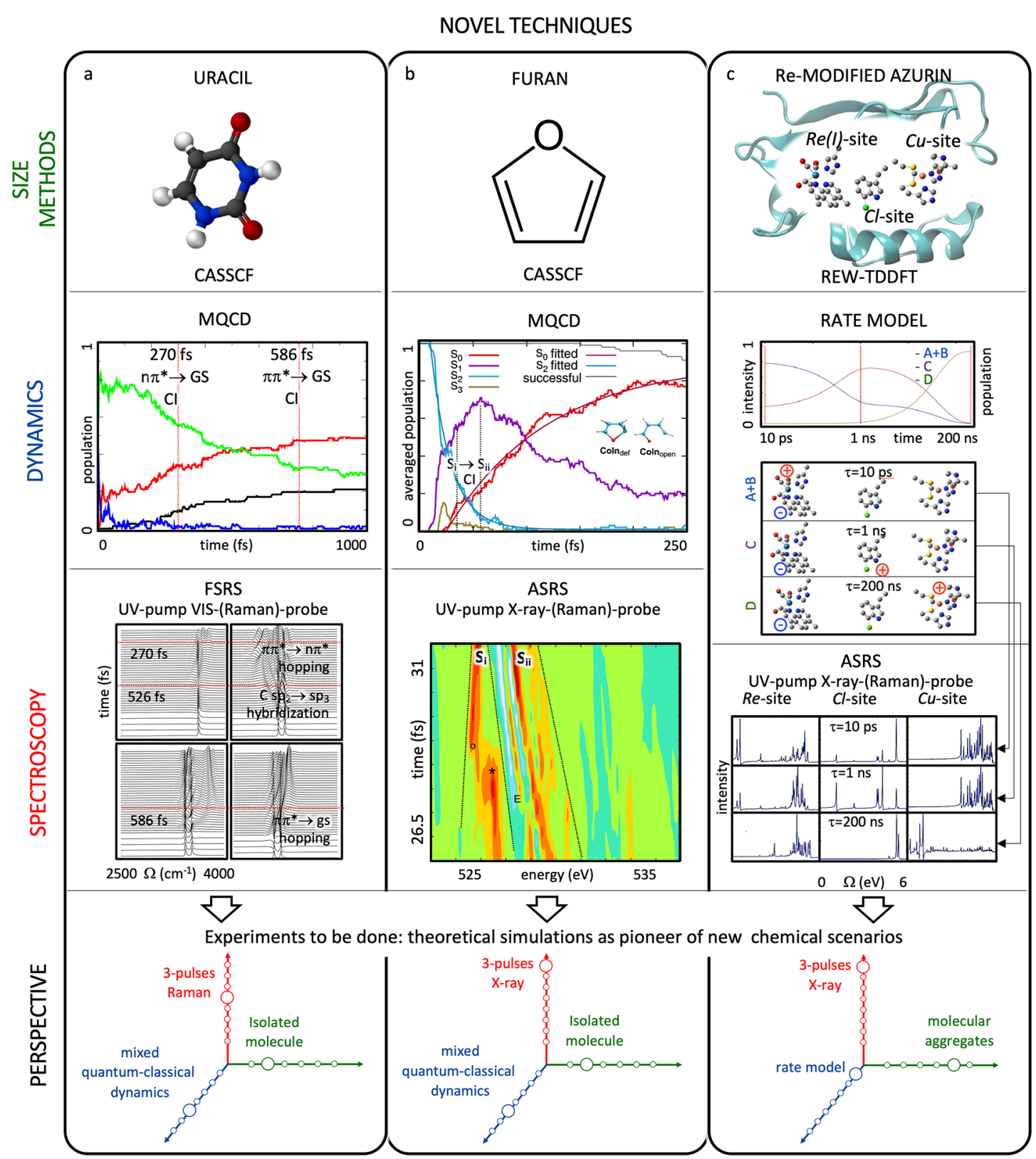

Figure 8. Three examples of novel spectroscopy simulation protocols of time-resolved vibrational and electronic Raman signal applied to (a) uracil (ref 63e), (b) furan (ref 89), and (c) azurin (ref 90). The state-of-the-art of the simulation in terms of system size/electronic structure method, molecular dynamics protocol, and spectroscopy technique are shown in the schematic representation of the three Grand Challenges (see Figure 5). An extended caption for this figure with additional details can be found in the Supporting Information. Figures rights: (a) Adapted with permission from ref 63e. Copyright 2014 ACS. (b) Adapted from ref 89, licensed under a Creative Commons Attribution (CC BY) license. (c) Adapted with permission from ref 90.

\subsubsection{Femtosecond Stimulated Raman Scattering (FSRS).}

${ }^{88}$ FSRS is a three-pulse experiment in which the dynamics of a molecule is initiated by an actinic pulse and the evolution of the vibrational spectrum is probed by stimulated Raman scattering (SRS), with a Raman sequence consisting of a picosecond Raman pump synchronized with a femtosecond Stokes pulse. In a first application, a simulation protocol was reported that combined non-adiabatic on-the-fly $\mathrm{MD}$ at the CASSCF level of theory with a mode-tracking algorithm to obtain the instantaneous frequencies of high-frequency spectator modes $(\mathrm{C}-\mathrm{H}$ and $\mathrm{N}-\mathrm{H}$ stretching) of photoexcited uracil (see Figure $8 \mathrm{a})^{63 \mathrm{e}}$ and demonstrated that the FSRS signal is sensitive to the non-adiabatic relaxation events in the vicinity of CIs. The experimental implementation of this technique could help determine the relative importance of the $\pi \pi^{*} \rightarrow$ ground state and the $\pi \pi^{*} \rightarrow \mathrm{n} \pi^{*}$ deactivation channels in DNA/RNA, still a matter of intense debate.

3.3.2. Attosecond Stimulated Raman Spectroscopy (ASRS). ASRS extends the ideas of FSRS to the attosecond time domain by superimposing a femtosecond Raman pump pulse with an attosecond Stokes pulse. The ASRS signals are highly sensitive to electronic coherences either created by the inelastic Raman process (resonant with core-valence transitions) induced by the hybrid broad-narrow pulse sequence or emerging from the off-diagonal elements of the density matrix which become different from zero in the case of non-adiabatic events such as the passage through or close to a CI. As the temporal and spectral resolution of the proposed experiment 
are not Fourier-conjugate pairs, this setup can monitor the evolution of the coherences with an attosecond temporal resolution without sacrificing spectral resolution.

The ability of ASRS to monitor the few femtoseconds short passage through a CI has been theoretically demonstrated on the ring-opening of furan (see Figure $8 \mathrm{~b}$ ). ${ }^{89}$ ASRS signals were simulated by on-the-fly $\mathrm{MD}$ at the CASSCF level in combination with oxygen core-valence calculations at the RASSCF level.

ASRS tracks coherences between valence states and is, therefore, particularly sensitive to variation of the electronic structure with the oxidation state. By probing valence dynamics via a core-excited intermediate, ASRS combines the spatial selectivity of X-ray spectroscopy with the much narrower linewidths of visible and UV spectra. ASRS in azurin was proposed for resolving long-range electron transfer, a central step in many biological redox reactions in living organisms (see Figure $8 \mathrm{c}) .{ }^{90}$ Using a simple kinetic model fitted to experimental lifetimes and coupled to restricted excitation window time-dependent density functional theory (REWTDDFT $)^{91}$ with the LC-PBE0 functional and conductor-like implicit solvent model (COSMO) to represent solvent effects, the authors demonstrated the higher sensitivity of ASRS in comparison to transient X-ray absorption spectroscopy.

\section{OPEN CHALLENGES}

\section{(... and revolutions)}

The past decade has witnessed remarkable progress in modeling transient spectroscopic signals of photoinduced events for large-size photoactive molecular systems in realistic conditions. This Perspective shows that computational spectroscopy has grown to its full maturity, going much beyond being a nice, but ancillary, complement of experiments. In many cases, it holds experimental accuracy, becoming a mandatory tool for the interpretation of complex multi-pulse spectroscopies. In other cases, simulations precede experimental capabilities and become drivers for future experimental developments.

Despite the many successes, there are still several outstanding problems within the three Grand Challenges, both theoretical and conceptual, which we briefly outline in this concluding section.

4.1. Electronic Structure Methods. 4.1.1. Benchmarking. A great effort will be needed in assessing the performance and understanding the advantages and limitations of the different methods by benchmarking them against established approaches (we refer to ref 92a for a nice essay on the subject). ${ }^{21 a, 92 b-g}$ This will require new codes and software interfaces (e.g., see SHARC, ${ }^{93}$ COBRAMM, ${ }^{93 b}$ NEWTON$\mathrm{X}^{93 \mathrm{c}}$ ) that allow various $\mathrm{QM}$ methods to be run on the same footing.

4.1.2. Size. We have shown (see Figure 7a) that the Frenkel exciton Hamiltonian is a convenient approach to model large and complex multi-chromophore systems and simulate their non-linear spectroscopy. However, this is based on the assumption that electronic excitations are (semi)localized on weakly interacting chromophores. Strongly interacting (and, possibly, reacting) units, displaying qualitatively different (possibly crossing), highly anharmonic ground- and excitedstate PESs (as for instance DNA/RNA oligomers ${ }^{94}$ ), are still challenging to describe. A promising strategy is to marry the brute-force approach of increasing the size of the QM region to the excitonic one by the use of larger, but still computationally manageable, QM building blocks for the exciton Hamiltonian, which embrace a set of strongly interacting chromophores. This would make it possible to describe the photoinduced processes (including delocalized and charge-transfer states), still concurrently accounting for the interactions among the selected QM regions as described by the exciton Hamiltonian and thus leveraging the intrinsic parallelizability of the calculations of its terms that is suitable for modern multicore architectures and GPU accelerated algorithms.

4.1.3. Machine Learning (ML). Deeply integrated QM and ML methods are expected to disclose new practical approximate ways to speed up electronic structure calculations. For instance, ML could help in avoiding the explicit estimate of the many expensive, but negligible, bottleneck terms in multiconfigurational and perturbative QM methods, still retaining their accuracy. Development and benchmarking of these approaches will be needed to prove their robustness and general applicability (see ref 95).

4.1.4. Quantum Computing. Driven by the main computing market leaders as well as new players (e.g., IBM, Google, Microsoft, D-Wave, etc.), the quest for quantum supremacy has already led to prototype CPUs based on different and competing quantum bit architectures. This makes the idea of modeling a quantum system by a quantum computer more than a dream (e.g., refs 96a-c). Notably, first applications and software packages for quantum computing simulations have just appeared (e.g., OpenFermion), ${ }^{97}$ with IBM already opening its quantum computing platform to the scientific community, thus making this one of the most attractive directions for the future.

4.2. Non-adiabatic Molecular Dynamics. 4.2.1. TSH in the Exciton Picture. The strength of the exciton Hamiltonian approach is that it can be coupled to a TSH non-adiabatic dynamics scheme, such as the Tully's FSSH. This requires updating the parameters of the electronic Hamiltonian on-thefly, as the nuclear configuration evolves in time, by computing at each step the derivatives of the exciton model parameters with respect to the nuclear coordinates, and mapping these and the NACs. ${ }^{3 f}$ In aggregates, due to the many close-lying excited states, new problems arise as one needs to either compute many NACs at each step of the propagation (strongly coupled chromophores) or deal with trivial crossings (non-interacting chromophores in the aggregate). This is a very promising strategy, as recently shown by a few preliminary works. ${ }^{3 e, 53}$ The study of the photophysics of DNA/RNA (a test case for flexible strongly coupled and reacting multi-chromophoric systems) will provide an excellent playground for these developments.

4.2.2. ML and Classical Force Fields for Excited States. Developments of atomistic ML tools, based on transfer learning on gold standard datasets optimally spanning the physics of interest, promise to deliver with low computational cost PESs as accurate as those obtained from the most expensive QM methods. ML approaches can also be used to produce, by a trained and driven re-parametrization of classical FFs, low-cost and accurate analytical PESs for the excited states, thus allowing non-adiabatic MD to be performed at the cost of classical MD. Recent results and proofs of concept are very promising. ${ }^{98 a-c}$ An alternative technique for obtaining coupled high-dimensional PESs is the anchor points reactive potential (APRP) method, which describes few largeamplitude (reactive) modes explicitly based on QM calculations, whereas small-amplitude (secondary) modes are 
described in MM-like terms. ${ }^{36 c, 98 d}$ Overall, these methods are expected to speed up MD and improve the accuracy by delivering optimal PESs.

4.3. Theoretical Spectroscopy. Future developments in theoretical spectroscopy are intimately linked to the corresponding advances in experimental techniques, enabled by either novel light sources (such as ultrashort coherent X-rays) or novel methodologies (such as ultrafast spectroscopy with quantum light).

4.3.1. Multi-Dimensional Extensions. Multi-dimensional extensions of some of the presented novel techniques (e.g., 2DFSRS $)^{99}$ would provide access to anharmonicities and intermode couplings in excited states that are influenced by the coupling in the vicinity of a CI. ${ }^{3 \mathrm{~g}}$

4.3.2. Time-Resolved Diffraction. Time-resolved diffraction with ultrashort X-ray or electron pulses has the advantage to directly encode spatial information, revealing the location of the created coherences: ${ }^{3 g}$ data interpretation will need accurate modeling of these signals. ${ }^{100}$

4.3.3. Simplified Tools. Simplified tools need to be made available to experimentalists and non-expert users to assist spectroscopic characterization of molecular materials beyond the academic lab. The development of black-box tools for spectral analysis and interpretation, and their integration within a productive environment, would be a major achievement, calling for a standardization of the language through the development of a common ontology in materials modeling. ${ }^{101}$

\section{CONCLUSION}

In summary, to achieve a complete and accurate description of the complex transient spectroscopy in large size chromophores and multi-chromophoric systems, major challenges are still in the way. Nonetheless, the smart integration of classical and quantum chemical (static and dynamic) approaches and the improvements in exciton Hamiltonian schemes, combined with the development in high-performance multi-core architectures, GPU accelerated algorithms, and machine learning techniques, draw a clear and promising path. Finally, the emerging field of quantum computing with the first example of quantum supremacy just appeared, ${ }^{102}$ which seems to announce the dawn of a new era, painting an even more optimistic horizon. This revolution will likely lead to a disruptive change in the high-performance computing world as we know it, heralding a plot twist in Quantum Chemistry Tales.

\section{ASSOCIATED CONTENT}

\section{SI Supporting Information}

The Supporting Information is available free of charge at https://pubs.acs.org/doi/10.1021/jacs.0c04952.

Extended versions of the figure captions and related references (PDF)

\section{AUTHOR INFORMATION}

\section{Corresponding Authors}

Artur Nenov - Dipartimento di Chimica Industriale, Università degli Studi di Bologna, I-40136 Bologna, Italy; ำ orcid.org/ 0000-0003-3071-5341; Email: artur.nenov@unibo.it

Marco Garavelli - Dipartimento di Chimica Industriale, Università degli Studi di Bologna, I-40136 Bologna, Italy; (1) orcid.org/0000-0002-0796-289X;

Email: marco.garavelli@unibo.it

\section{Authors}

Irene Conti - Dipartimento di Chimica Industriale, Università degli Studi di Bologna, I-40136 Bologna, Italy; 이이.org/ 0000-0001-7982-4480

Giulio Cerullo - Dipartimento di Fisica, Politecnico di Milano, IFN-CNR, I-20133 Milano, Italy; 이이.org/0000-00029534-2702

Complete contact information is available at:

https://pubs.acs.org/10.1021/jacs.0c04952

\section{Notes}

The authors declare no competing financial interest.

\section{ACKNOWLEDGMENTS}

The authors acknowledge support from the H2020-NMBPTO-IND-2018-2020/DT-NMBP-09-2018 project SIMDOME, Grant agreement No. 814492. M.G. and G.C. acknowledge support from the European Research Council Advanced Grant STRATUS (ERC2011AdG No. 291198). Financial support from the PHANTOMS project, PRIN: PROGETTI DI RICERCA DI RILEVANTE INTERESSE NAZIONALE Bando 2017, Prot. 2017A4XRCA, is acknowledged. G.C. acknowledges support from the PRIN 2017 project 201795SBA3 - HARVEST. We thank Francesco Segatta for the useful discussions.

\section{ABBREVIATIONS}

2D, two-dimensional; 2DES, two-dimensional electronic spectroscopy; 2DIR, two-dimensional infrared; ADC, algebraic diagrammatic construction; AIFDEM, ab initio FrenkelDavydov exciton model; AIMC, ab initio multiple cloning; AIMS, ab initio multiple spawning; APRP, anchor points reactive potential; ASCI, adaptive sampling configuration interaction; ASRS, attosecond stimulated Raman scattering; CASSCF, complete active space self-consistent field; CASPT2, complete active space second-order perturbation theory; CC, coupled cluster; $\mathrm{CC} 2$, coupled-cluster singles and doubles; CGF, cumulant expansion of Gaussian fluctuations; CI, conical intersection; CIS, configuration interaction singles; COSMO, conductor-like screening model; CT-MQC, coupled-trajectory mixed quantum-classical; DFT, density functional theory; DHO, displaced harmonic oscillator; DISH-XF, decoherenceinduced surface hopping based on exact factorization; DMRG, density matrix renormalization group; DOF, degrees of freedom; $\mathrm{DQ}$ dipole and quadrupole moments; EF, exact factorization formalism; EMMO, European materials modeling ontology release; EOM-CC, equation-of-motion coupled cluster; EOM-SF-CC, equation-of-motion spin-flip coupled cluster; ESA, excited-state absorption; FCIQMC, full configuration interaction quantum Monte Carlo; FF, force field; FSRS, femtosecond stimulated Raman scattering; FSSH, fewest switches surface hopping; GDR, dephasing representation in Gaussian basis; GASPT2, generalized active space secondorder perturbation theory; GASSCF, generalized active space self-consistent field; GPU, graphic processing unit for dataparallel computing; HCI, heat-bath configuration interaction; HEOM, hierarchical equations of motion; HOOP, hydrogen out-of-plane; i-QUAPI, iterative quasi-adiabatic propagator path integral; LH2, light-harvesting complex 2; LR, linear response; LR-CC, linear-response coupled cluster; MC, Monte Carlo; MC-PDFT, multi-configurational pair density functional theory; MCTDH, multi-configurational time-dependent Har- 
tree; MD, molecular dynamics; ML, machine learning; MQCD, mixed quantum-classical dynamics; MR-ADC, multireference algebraic diagrammatic construction; MR-CC, multireference coupled cluster; $\mathrm{MRCI}$, multi-reference configuration interaction; MRMP2, multi-reference second-order MøllerPlesset perturbation theory; NAC, non-adiabatic coupling; NanoDOME, nanomaterials via gas-phase synthesis: a designoriented modeling and engineering approach, $\mathrm{H} 2020$ project; NEVPT2, N-Electron valence state second-order perturbation theory; ORMAS, occupation restricted multiple active space; OWVP, outgoing wave variational principle; PES, potential energy surfaces; PLDM, partial linearized density matrix; PW, pseudo-wavefunction; QD, quantum dynamics; QM, quantum mechanics; QMC, quantum Monte Carlo; QM/MM, quantum mechanics/molecular mechanics; RASPT2, restricted active space second-order perturbation theory; RASSCF, restricted active space self-consistent field; REKS, spin-restricted ensemble-referenced Kohn-Sham; REW-TDDFT, restricted excitation window time-dependent density functional theory; RMProp, R-matrix propagation; RT, real-time; SA-SF-DFT, spin-adapted spin-flip density functional theory; SCF, selfconsistent field; SC-SF-ORMAS, spin-complete spin-flip occupation restricted multiple active space; SE, stimulated emission; SF-ADC, spin-flip algebraic diagrammatic construction; SF-CIS, spin-flip configuration interaction singles; SFDFT, spin-flip density functional theory; SimDOME, digital ontology-based modeling environment for simulation of materials, H2020 project; SI-SA-REKS, state-interaction with state-average spin-restricted ensemble-referenced KohnSham; SOS, sum over all states; SR-CI, single-reference configuration interaction; TA, transient absorption; TDDFT, time-dependent density functional theory; TD-DMRG, timedependent density matrix renormalization group; TDM, transition dipole moment; TRPES, time-resolved photoelectron spectroscopy; TRUECARS, transient redistribution of ultrafast electronic coherences in attosecond Raman signals; $\mathrm{TSH}$, trajectory surface hopping; v2RDM, variational twoelectron reduced-density matrix; vMCG, variational MultiConfigurational Gaussian wavepacket; WF, wavefunction; XMS-CASPT2, extended multi-state complete active-space second-order perturbation theory

\section{REFERENCES}

(1) (a) Norrish, R. G. W.; Porter, G. Chemical reactions produced by very high light intensities. Nature 1949, 164 (4172), 658-658. (b) Porter, G.-N. Flash photolysis and spectroscopy. A new method for the study of free radical reactions. Proc. R. Soc. London Ser. A 1950, 200 (1061), 284-300.

(2) (a) Maiuri, M.; Garavelli, M.; Cerullo, G. Ultrafast Spectroscopy: state-of-the-art and Open Challenges. J. Am. Chem. Soc. 2020, 142 (1), 3-15. (b) Elsaesser, T. Introduction: Ultrafast Processes in Chemistry. Chem. Rev. 2017, 117, 10621-10622.

(3) (a) Crespo-Otero, R.; Barbatti, M. Recent Advances and Perspectives on Nonadiabatic Mixed Quantum-Classical Dynamics. Chem. Rev. 2018, 118 (15), 7026-7068. (b) Nelson, T. R.; White, A. J.; Bjorgaard, J. A.; Sifain, A. E.; Zhang, Y.; Nebgen, B.; FernandezAlberti, S.; Mozyrsky, D.; Roitberg, A. E.; Tretiak, S. Non-adiabatic Excited-State Molecular Dynamics: Theory and Applications for Modeling Photophysics in Extended Molecular Materials. Chem. Rev. 2020, 120 (4), 2215-2287. (c) Mai, S.; González, L. Molecular Photochemistry: Recent Developments in Theory. Angew. Chem., Int. Ed. 2020, DOI: 10.1002/anie.201916381. (d) Curchod, B. F. E.; Martínez, T. J. Ab Initio Nonadiabatic Quantum Molecular Dynamics. Chem. Rev. 2018, 118 (7), 3305-3336. (e) Sisto, A.; Glowacki, D. R.; Martinez, T. J. Ab Initio Nonadiabatic Dynamics of Multi- chromophore Complexes: A Scalable Graphical-Processing-UnitAccelerated Exciton Framework. Acc. Chem. Res. 2014, 47 (9), 2857-2866. (f) Segatta, F.; Cupellini, L.; Garavelli, M.; Mennucci, B. Quantum Chemical Modeling of the Photoinduced Activity of Multichromophoric Biosystems: Focus Review. Chem. Rev. 2019, 119 (16), 9361-9380. (g) Kowalewski, M.; Fingerhut, B. P.; Dorfman, K. E.; Bennett, K.; Mukamel, S. Simulating coherent multidimensional spectroscopy of nonadiabatic molecular processes: From the infrared to the x-ray regime. Chem. Rev. 2017, 117 (19), 12165-12226. (h) Borrego-Varillas, R.; Nenov, A.; Ganzer, L.; Oriana, A.; Manzoni, C.; Tolomelli, A.; Rivalta, I.; Mukamel, S.; Garavelli, M.; Cerullo, G. Two-dimensional UV spectroscopy: a new insight into the structure and dynamics of biomolecules. Chemical Science 2019, 10 (43), 9907-9921. (i) Segarra-Marti, J.; Mukamel, S.; Garavelli, M.; Nenov, A.; Rivalta, I. Towards Accurate Simulation of Two-Dimensional Electronic Spectroscopy. Top Curr. Chem. (Z) 2018, 376 (3), 24. (j) Zhang, Y.; Hua, W.; Bennett, K.; Mukamel, S. Nonlinear Spectroscopy of Core and Valence Excitations Using Short X-Ray Pulses: Simulation Challenges. Top. Curr. Chem. 2014, 368, 273-345. (k) Mitríc, R.; Petersen, j.; Bonačić-Koutecký, V. Multistate Nonadiabatic Dynamics "on the Fly" in Complex Systems and Its Control by Laser Fieldes. Conical Intersections: Theory, Computation and Experiment 2011, 17, 497-568.

(4) (a) Andrade, X.; Alberdi-Rodriguez, J.; Strubbe, D. A.; Oliveira, M. J. T.; Nogueira, F.; Castro, A.; Muguerza, J.; Arruabarrena, A.; Louie, S. G.; Aspuru-Guzik, A.; Rubio, A.; Marques, M. A. L. Timedependent density-functional theory in massively parallel computer architectures: the octopus project. J. Phys.: Condens. Matter 2012, 24 (23), 233202. (b) Peng, C.; Calvin, J. A.; Pavosevic, F.; Zhang, J.; Valeev, E. F. Massively Parallel Implementation of Explicitly Correlated Coupled-Cluster Singles and Doubles Using TiledArray Framework. J. Phys. Chem. A 2016, 120 (51), 10231-10244. (c) Hohenstein, E. G.; Luehr, N.; Ufimtsev, I. S.; Martinez, T. J. An atomic orbital-based formulation of the complete active space selfconsistent field method on graphical processing units. J. Chem. Phys. 2015, 142 (22), 224103. (d) Snyder, J. W., Jr.; Fales, B. S.; Hohenstein, E. G.; Levine, B. G.; Martinez, T. J. A direct-compatible formulation of the coupled perturbed complete active space selfconsistent field equations on graphical processing units. J. Chem. Phys. 2017, 146 (17), 174113. (e) Snyder, J. W., Jr.; Hohenstein, E. G.; Luehr, N.; Martinez, T. J. An atomic orbital-based formulation of analytical gradients and nonadiabatic coupling vector elements for the state-averaged complete active space self-consistent field method on graphical processing units. J. Chem. Phys. 2015, 143 (15), 154107.

(5) (a) Aquilante, F.; Autschbach, J.; Carlson, R. K.; Chibotaru, L. F.; Delcey, M. G.; De Vico, L.; Fdez. Galvan, I.; Ferre, N.; Frutos, L. M.; Gagliardi, L.; Garavelli, M.; Giussani, A.; Hoyer, C. E.; Li Manni, G.; Lischka, H.; Ma, D.; Malmqvist, P. A.; Muller, T.; Nenov, A.; Olivucci, M.; Pedersen, T. B.; Peng, D.; Plasser, F.; Pritchard, B.; Reiher, M.; Rivalta, I.; Schapiro, I.; Segarra-Marti, J.; Stenrup, M.; Truhlar, D. G.; Ungur, L.; Valentini, A.; Vancoillie, S.; Veryazov, V.; Vysotskiy, V. P.; Weingart, O.; Zapata, F.; Lindh, R. Molcas 8: New capabilities for multiconfigurational quantum chemical calculations across the periodic table. J. Comput. Chem. 2016, 37 (5), 506-541. (b) Vogiatzis, K. D.; Ma, D.; Olsen, J.; Gagliardi, L.; de Jong, W. A. Pushing configuration-interaction to the limit: Towards massively parallel MCSCF calculations. J. Chem. Phys. 2017, 147 (18), 184111.

(6) (a) Shao, Y.; Gan, Z.; Epifanovsky, E.; Gilbert, A. T.; Wormit, M.; Kussmann, J.; Lange, A. W.; Behn, A.; Deng, J.; Feng, X.; et al. Advances in molecular quantum chemistry contained in the Q-Chem 4 program package. Mol. Phys. 2015, 113 (2), 184-215. (b) Turney, J. M.; Simmonett, A. C.; Parrish, R. M.; Hohenstein, E. G.; Evangelista, F. A.; Fermann, J. T.; Mintz, B. J.; Burns, L. A.; Wilke, J. J.; Abrams, M. L.; et al. Psi4: an open-source ab initio electronic structure program. Wiley Interdiscip. Rev.: Comput. Mol. Sci. 2012, 2 (4), 556-565. (c) Luehr, N.; Jin, A. G.; Martinez, T. J. Ab initio interactive molecular dynamics on graphical processing units (GPUs). J. Chem. Theory Comput. 2015, 11 (10), 4536-44. (d) Peng, C.; Lewis, C.; Wang, X.; Clement, M.; Pavosevic, F.; Zhang, J.; Rishi, V.; 
Teke, N.; Pierce, K.; Calvin, J.; Kenny, J.; Seidl, E.; Janssen, C.; Valeev, E. The Massively Parallel Quantum Chemistry Program (MPQC), Version 4.0.0-beta.1; http://github.com/ValeevGroup/ mpqc. (e) Valiev, M.; Bylaska, E.J.; Govind, N.; Kowalski, K.; Straatsma, T.P.; Van Dam, H.J.J.; Wang, D.; Nieplocha, J.; Apra, E.; Windus, T.L.; de Jong, W.A. NWChem: A comprehensive and scalable open-source solution for large scale molecular simulations. Comput. Phys. Commun. 2010, 181 (9), 1477-1489.

(7) Aquilante, F.; Delcey, M. G.; Pedersen, T. B.; Fdez. Galván, I.; Lindh, R. Inner projection techniques for the low-cost handling of two-electron integrals in quantum chemistry. Mol. Phys. 2017, 115 (17-18), 2052-2064.

(8) Sun, Q.; Yang, J.; Chan, G. K.-L. A general second order complete active space self-consistent-field solver for large-scale systems. Chem. Phys. Lett. 2017, 683, 291-299.

(9) (a) Wouters, S.; Poelmans, W.; Ayers, P. W.; Van Neck, D. CheMPS2: A free open-source spin-adapted implementation of the density matrix renormalization group for ab initio quantum chemistry. Comput. Phys. Commun. 2014, 185 (6), 1501-1514. (b) Yanai, T.; Saitow, M.; Xiong, X. G.; Chalupsky, J.; Kurashige, Y.; Guo, S.; Sharma, S. Multistate Complete-Active-Space Second-Order Perturbation Theory Based on Density Matrix Renormalization Group Reference States. J. Chem. Theory Comput. 2017, 13 (10), 4829-4840. (c) Guo, S.; Watson, M. A.; Hu, W.; Sun, Q.; Chan, G. K.-L. Nelectron valence state perturbation theory based on a density matrix renormalization group reference function, with applications to the chromium dimer and a trimer model of poly (p-phenylenevinylene). J. Chem. Theory Comput. 2016, 12 (4), 1583-1591. (d) Saitow, M.; Kurashige, Y.; Yanai, T. Multireference configuration interaction theory using cumulant reconstruction with internal contraction of density matrix renormalization group wave function. J. Chem. Phys. 2013, 139 (4), 044118. (e) Li Manni, G.; Smart, S. D.; Alavi, A. Combining the Complete Active Space Self-Consistent Field Method and the Full Configuration Interaction Quantum Monte Carlo within a Super-CI Framework, with Application to Challenging MetalPorphyrins. J. Chem. Theory Comput. 2016, 12 (3), 1245-58. (f) Fosso-Tande, J.; Nguyen, T. S.; Gidofalvi, G.; DePrince, A. E. 3rd, Large-Scale Variational Two-Electron Reduced-Density-MatrixDriven Complete Active Space Self-Consistent Field Methods. J. Chem. Theory Comput. 2016, 12 (5), 2260-71. (g) Smith, J. E. T.; Mussard, B.; Holmes, A. A.; Sharma, S. Cheap and Near Exact CASSCF with Large Active Spaces. J. Chem. Theory Comput. 2017, 13 (11), 5468-5478. (h) Levine, D. S.; Hait, D.; Tubman, N. M.; Lehtola, S.; Whaley, K. B.; Head-Gordon, M. CASSCF with Extremely Large Active Spaces using the Adaptive Sampling Configuration Interaction Method. J. Chem. Theory Comput. 2020, 16 (4), 2340-2354.

(10) (a) Ma, D.; Li Manni, G.; Olsen, J.; Gagliardi, L. Second-Order Perturbation Theory for Generalized Active Space Self-ConsistentField Wave Functions. J. Chem. Theory Comput. 2016, 12 (7), 320813. (b) Ivanic, J. Direct configuration interaction and multiconfigurational self-consistent-field method for multiple active spaces with variable occupations. I. Method. J. Chem. Phys. 2003, 119 (18), 9364-9376. (c) Choe, Y.-K.; Nakao, Y.; Hirao, K. Multireference Møller-Plesset method with a complete active space configuration interaction reference function. J. Chem. Phys. 2001, 115 (2), 621-629.

(11) (a) Dreuw, A.; Head-Gordon, M. Single-reference ab initio methods for the calculation of excited states of large molecules. Chem. Rev. 2005, 105 (11), 4009-37. (b) Dreuw, A.; Wormit, M. The algebraic diagrammatic construction scheme for the polarization propagator for the calculation of excited states. Wiley Interdiscip. Rev.: Comput. Mol. Sci. 2015, 5 (1), 82-95. (c) Sneskov, K.; Christiansen, O. Excited state coupled cluster methods. Wiley Interdiscip. Rev.: Comput. Mol. Sci. 2012, 2 (4), 566-584. (d) Izsák, R. Single-reference coupled cluster methods for computing excitation energies in large molecules: The efficiency and accuracy of approximations. Wiley Interdiscip. Rev.: Comput. Mol. Sci. 2020, 10 (3), No. e1445. (e) Pinski, P.; et al. Sparse maps-A systematic infrastructure for reduced-scaling electronic structure methods. I. An efficient and simple linear scaling local MP2 method that uses an intermediate basis of pair natural orbitals. J. Chem. Phys. 2015, 143 (3), 034108. (f) Riplinger, C.; Pinski, P.; Becker, U.; Valeev, E. F.; Neese, F. Sparse maps-A systematic infrastructure for reduced-scaling electronic structure methods. II. Linear scaling domain based pair natural orbital coupled cluster theory. J. Chem. Phys. 2016, 144 (2), 024109.

(12) Gozem, S.; Melaccio, F.; Valentini, A.; Filatov, M.; HuixRotllant, M.; Ferre, N.; Frutos, L. M.; Angeli, C.; Krylov, A. I.; Granovsky, A. A.; Lindh, R.; Olivucci, M. Shape of Multireference, Equation-of-Motion Coupled-Cluster, and Density Functional Theory Potential Energy Surfaces at a Conical Intersection. J. Chem. Theory Comput. 2014, 10 (8), 3074-84.

(13) (a) Krylov, A. I. Spin-flip equation-of-motion coupled-cluster electronic structure method for a description of excited states, bond breaking, diradicals, and triradicals. Acc. Chem. Res. 2006, 39 (2), 8391. (b) Tsuchimochi, T. Spin-flip configuration interaction singles with exact spin-projection: Theory and applications to strongly correlated systems. J. Chem. Phys. 2015, 143 (14), 144114. (c) Manohar, P. U.; Krylov, A. I. A noniterative perturbative triples correction for the spin-flipping and spin-conserving equation-ofmotion coupled-cluster methods with single and double substitutions. J. Chem. Phys. 2008, 129 (19), 194105. (d) Lefrancois, D.; Tuna, D.; Martínez, T. J.; Dreuw, A. The spin-flip variant of the algebraicdiagrammatic construction yields the correct topology of S1/S0 conical intersections. J. Chem. Theory Comput. 2017, 13 (9), 44364441.

(14) Mato, J.; Gordon, M. S. A general spin-complete spin-flip configuration interaction method. Phys. Chem. Chem. Phys. 2018, 20 (4), 2615-2626.

(15) (a) Lyakh, D. I.; Musial, M.; Lotrich, V. F.; Bartlett, R. J. Multireference nature of chemistry: the coupled-cluster view. Chem. Rev. 2012, 112 (1), 182-243. (b) Kovyrshin, A.; Neugebauer, J. Analytical gradients for excitation energies from frozen-density embedding. Phys. Chem. Chem. Phys. 2016, 18 (31), 20955-20975. (c) Sokolov, A. Y. Multi-reference algebraic diagrammatic construction theory for excited states: General formulation and first-order implementation. J. Chem. Phys. 2018, 149 (20), 204113.

(16) Lischka, H.; Nachtigallova, D.; Aquino, A. J.; Szalay, P. G.; Plasser, F.; Machado, F. B.; Barbatti, M. Multireference approaches for excited states of molecules. Chem. Rev. 2018, 118 (15), 72937361.

(17) Maitra, N. T. Charge transfer in time-dependent density functional theory. J. Phys.: Condens. Matter 2017, 29 (42), 423001.

(18) Levine, B. G.; Ko, C.; Quenneville, J.; MartÍnez, T. J. Conical intersections and double excitations in time-dependent density functional theory. Mol. Phys. 2006, 104 (5-7), 1039-1051.

(19) Elliott, P.; Goldson, S.; Canahui, C.; Maitra, N. T. Perspectives on double-excitations in TDDFT. Chem. Phys. 2011, 391 (1), 110119.

(20) Tawada, Y.; Tsuneda, T.; Yanagisawa, S.; Yanai, T.; Hirao, K. A long-range-corrected time-dependent density functional theory. $J$. Chem. Phys. 2004, 120 (18), 8425-33.

(21) (a) Li, H.; Nieman, R.; Aquino, A. J.; Lischka, H.; Tretiak, S. Comparison of LC-TDDFT and ADC (2) methods in computations of bright and charge transfer states in stacked oligothiophenes. $J$. Chem. Theory Comput. 2014, 10 (8), 3280-3289. (b) Verma, P.; Truhlar, D. G. Status and Challenges of Density Functional Theory. Trends in Chemistry 2020, 2 (4), 302-318. (c) Verma, P.; Wang, Y.; Ghosh, S.; He, X.; Truhlar, D. G. Revised M11 exchange-correlation functional for electronic excitation energies and ground-state properties. J. Phys. Chem. A 2019, 123 (13), 2966-2990.

(22) (a) Herbert, J. M.; Zhang, X.; Morrison, A. F.; Liu, J. Beyond time-dependent density functional theory using only single excitations: Methods for computational studies of excited states in complex systems. Acc. Chem. Res. 2016, 49 (5), 931-941. (b) Zhang, X.; Herbert, J. M. Spin-flip, tensor equation-of-motion configuration interaction with a density-functional correction: A spin-complete method for exploring excited-state potential energy surfaces. J. Chem. Phys. 2015, 143 (23), 234107. (c) Marian, C. M.; Heil, A.; 
Kleinschmidt, M. The DFT/MRCI method. Wiley Interdiscip. Rev.: Comput. Mol. Sci. 2019, 9 (2), No. e1394.

(23) (a) Li, Z.; Liu, W. First-order nonadiabatic coupling matrix elements between excited states: A Lagrangian formulation at the CIS, RPA, TD-HF, and TD-DFT levels. J. Chem. Phys. 2014, 141 (1), 014110. (b) Shu, Y.; Parker, K. A.; Truhlar, D. G. Dual-functional Tamm-Dancoff approximation: a convenient density functional method that correctly describes $S_{1} / S_{0}$ conical intersections. J. Phys. Chem. Lett. 2017, 8 (10), 2107-2112.

(24) (a) Hedin, L. New method for calculating the one-particle Green's function with application to the electron-gas problem. Phys. Rev. 1965, 139 (3A), A796. (b) Blase, X.; Duchemin, I.; Jacquemin, D. The Bethe-Salpeter equation in chemistry: relations with TDDFT, applications and challenges. Chem. Soc. Rev. 2018, 47 (3), 1022-1043. (c) Salpeter, E. E.; Bethe, H. A. A relativistic equation for bound-state problems. Phys. Rev. 1951, 84 (6), 1232.

(25) Gräfenstein, J.; Cremer, D. Can density functional theory describe multi-reference systems? Investigation of carbenes and organic biradicals. Phys. Chem. Chem. Phys. 2000, 2 (10), 2091-2103. (26) (a) Ullrich, C.; Kohn, W. Kohn-Sham theory for ground-state ensembles. Phys. Rev. Lett. 2001, 87 (9), 093001. (b) Filatov, M. Spinrestricted ensemble-referenced Kohn-Sham method: basic principles and application to strongly correlated ground and excited states of molecules. Wiley Interdiscip. Rev.: Comput. Mol. Sci. 2015, 5 (1), 146167.

(27) (a) Wesolowski, T. A.; Shedge, S.; Zhou, X. Frozen-Density Embedding Strategy for Multilevel Simulations of Electronic Structure. Chem. Rev. 2015, 115 (12), 5891-928. (b) Daday, C.; Konig, C.; Neugebauer, J.; Filippi, C. Wavefunction in density functional theory embedding for excited states: which wavefunctions, which densities? ChemPhysChem 2014, 15 (15), 3205-17. (c) Daday, C.; Konig, C.; Valsson, O.; Neugebauer, J.; Filippi, C. State-Specific Embedding Potentials for Excitation-Energy Calculations. J. Chem. Theory Comput. 2013, 9 (5), 2355-67. (d) Dresselhaus, T.; Neugebauer, J.; Knecht, S.; Keller, S.; Ma, Y.; Reiher, M. Selfconsistent embedding of density-matrix renormalization group wavefunctions in a density functional environment. J. Chem. Phys. 2015, 142 (4), 044111. (e) Hofener, S.; Visscher, L. Wave Function Frozen-Density Embedding: Coupled Excitations. J. Chem. Theory Comput. 2016, 12 (2), 549-57. (f) Prager, S.; Zech, A.; Aquilante, F.; Dreuw, A.; Wesolowski, T. A. First time combination of frozen density embedding theory with the algebraic diagrammatic construction scheme for the polarization propagator of second order. $J$. Chem. Phys. 2016, 144 (20), 204103.

(28) (a) Gagliardi, L.; Truhlar, D. G.; Li Manni, G.; Carlson, R. K.; Hoyer, C. E.; Bao, J. L. Multiconfiguration Pair-Density Functional Theory: A New Way To Treat Strongly Correlated Systems. Acc. Chem. Res. 2017, 50 (1), 66-73. (b) Sharma, P.; Bernales, V.; Knecht, S.; Truhlar, D. G.; Gagliardi, L. Density matrix renormalization group pair-density functional theory (DMRG-PDFT): singlet-triplet gaps in polyacenes and polyacetylenes. Chemical science 2019, 10 (6), 1716-1723. (c) Mostafanejad, M.; DePrince, A. E. 3rd, Combining Pair-Density Functional Theory and Variational Two-Electron Reduced-Density Matrix Methods. J. Chem. Theory Comput. 2019, 15 (1), 290-302.

(29) Ghosh, S.; Verma, P.; Cramer, C. J.; Gagliardi, L.; Truhlar, D. G. Combining Wave Function Methods with Density Functional Theory for Excited States. Chem. Rev. 2018, 118 (15), 7249-7292.

(30) Brunk, E.; Rothlisberger, U. Mixed Quantum Mechanical/ Molecular Mechanical Molecular Dynamics Simulations of Biological Systems in Ground and Electronically Excited States. Chem. Rev. 2015, 115 (12), 6217-63.

(31) Vreven, T.; Morokuma, K. Hybrid methods: Oniom (qm: mm) and $\mathrm{qm} / \mathrm{mm}$. Annu. Rep. Comput. Chem. 2006, 2, 35-51.

(32) (a) Caprasecca, S.; Curutchet, C.; Mennucci, B. Toward a Unified Modeling of Environment and Bridge-Mediated Contributions to Electronic Energy Transfer: A Fully Polarizable QM/MM/ PCM Approach. J. Chem. Theory Comput. 2012, 8 (11), 4462-73. (b) Boulanger, E.; Thiel, W. Toward QM/MM Simulation of
Enzymatic Reactions with the Drude Oscillator Polarizable Force Field. J. Chem. Theory Comput. 2014, 10 (4), 1795-809. (c) Shi, Y.; Ren, P.; Schnieders, M.; Piquemal, J.-P. Polarizable force fields for biomolecular modeling. In Reviews in computational chemistry; Parrill, A. L., Lipkowitz, K. B., Eds.; Wiley, 2015; Vol. 28, p 51. (d) Loco, D.; Lagardere, L.; Caprasecca, S.; Lipparini, F.; Mennucci, B.; Piquemal, J. P. Hybrid QM/MM Molecular Dynamics with AMOEBA Polarizable Embedding. J. Chem. Theory Comput. 2017, 13 (9), 4025-4033. (e) Sahoo, S. K.; Nair, N. N. Interfacing the Core-Shell or the Drude Polarizable Force Field With Car-Parrinello Molecular Dynamics for QM/MM Simulations. Front. Chem. 2018, 6, 275.

(33) Reinholdt, P.; Kongsted, J.; Olsen, J. M. H. Polarizable density embedding: A solution to the electron spill-out problem in multiscale modeling. J. Phys. Chem. Lett. 2017, 8 (23), 5949-5958.

(34) Segarra-Marti, J.; Segatta, F.; Mackenzie, T. A.; Nenov, A.; Rivalta, I.; Bearpark, M. J.; Garavelli, M. Modeling multidimensional spectral lineshapes from first principles: application to water-solvated adenine. Faraday Discuss. 2020, 221 (0), 219-244.

(35) (a) Morrison, A. F.; You, Z. Q.; Herbert, J. M. Ab Initio Implementation of the Frenkel-Davydov Exciton Model: A Naturally Parallelizable Approach to Computing Collective Excitations in Crystals and Aggregates. J. Chem. Theory Comput. 2014, 10 (12), 5366-76. (b) Li, X.; Parrish, R. M.; Liu, F.; Kokkila Schumacher, S. I.; Martínez, T. J. An ab initio exciton model including charge-transfer excited states. J. Chem. Theory Comput. 2017, 13 (8), 3493-3504.

(36) (a) Nakamura, H.; Truhlar, D. G. Direct diabatization of electronic states by the fourfold way. II. Dynamical correlation and rearrangement processes. J. Chem. Phys. 2002, 117 (12), 5576-5593. (b) Xu, X.; Zheng, J.; Yang, K. R.; Truhlar, D. G. Photodissociation dynamics of phenol: Multistate trajectory simulations including tunneling. J. Am. Chem. Soc. 2014, 136 (46), 16378-16386. (c) Li, S. L.; Truhlar, D. G. Full-dimensional ground-and excited-state potential energy surfaces and state couplings for photodissociation of thioanisole. J. Chem. Phys. 2017, 146 (6), 064301. (d) Voityuk, A. A.; Rösch, N. Fragment charge difference method for estimating donoracceptor electronic coupling: Application to DNA $\pi$-stacks. J. Chem. Phys. 2002, 117 (12), 5607-5616. (e) Hsu, C.-P.; You, Z.-Q.; Chen, H.-C. Characterization of the short-range couplings in excitation energy transfer. J. Phys. Chem. C 2008, 112 (4), 1204-1212. (f) Subotnik, J. E.; Yeganeh, S.; Cave, R. J.; Ratner, M. A. Constructing diabatic states from adiabatic states: extending generalized Mulliken-Hush to multiple charge centers with boys localization. J. Chem. Phys. 2008, 129 (24), 244101. (g) Subotnik, J. E.; Alguire, E. C.; Ou, Q.; Landry, B. R.; Fatehi, S. The requisite electronic structure theory to describe photoexcited nonadiabatic dynamics: nonadiabatic derivative couplings and diabatic electronic couplings. Acc. Chem. Res. 2015, 48 (5), 1340-50. (h) Hoyer, C. E.; Xu, X.; Ma, D.; Gagliardi, L.; Truhlar, D. G. Diabatization based on the dipole and quadrupole: the DQ method. J. Chem. Phys. 2014, 141 (11), 114104.

(37) (a) Conwell, E. M.; McLaughlin, P. M.; Bloch, S. M. Chargetransfer excitons in DNA. J. Phys. Chem. B 2008, 112 (7), 2268-72. (b) Blancafort, L.; Voityuk, A. A. Exciton delocalization, charge transfer, and electronic coupling for singlet excitation energy transfer between stacked nucleobases in DNA: an MS-CASPT2 study. J. Chem. Phys. 2014, 140 (9), 095102.

(38) (a) MacLeod, M. K.; Shiozaki, T. Communication: automatic code generation enables nuclear gradient computations for fully internally contracted multireference theory. J. Chem. Phys. 2015, 142 (5), 051103. (b) Park, J. W.; Shiozaki, T. Analytical Derivative Coupling for Multistate CASPT2 Theory. J. Chem. Theory Comput. 2017, 13 (6), 2561-2570. (c) Hu, W.; Chan, G. K.-L. Excited-state geometry optimization with the density matrix renormalization group, as applied to polyenes. J. Chem. Theory Comput. 2015, 11 (7), 30003009. (d) Maradzike, E.; Gidofalvi, G.; Turney, J. M.; Schaefer, H. F., 3rd; DePrince, A. E., 3rd Analytic Energy Gradients for Variational Two-Electron Reduced-Density-Matrix-Driven Complete Active Space Self-Consistent Field Theory. J. Chem. Theory Comput. 2017, 13 (9), 4113-4122. 
(39) (a) Tavernelli, I.; Curchod, B. F.; Laktionov, A.; Rothlisberger, $\mathrm{U}$. Nonadiabatic coupling vectors for excited states within timedependent density functional theory in the tamm-Dancoff approximation and beyond. J. Chem. Phys. 2010, 133 (19), 194104. (b) Plasser, F.; Crespo-Otero, R.; Pederzoli, M.; Pittner, J.; Lischka, H.; Barbatti, M. Surface Hopping Dynamics with Correlated SingleReference Methods: 9H-Adenine as a Case Study. J. Chem. Theory Comput. 2014, 10 (4), 1395-405. (c) Faraji, S.; Matsika, S.; Krylov, A. I. Calculations of non-adiabatic couplings within equation-ofmotion coupled-cluster framework: Theory, implementation, and validation against multi-reference methods. J. Chem. Phys. 2018, 148 (4), 044103. (d) Zhang, X.; Herbert, J. M. Analytic derivative couplings in time-dependent density functional theory: Quadratic response theory versus pseudo-wavefunction approach. J. Chem. Phys. 2015, 142 (6), 064109. (e) Alguire, E. C.; Ou, Q.; Subotnik, J. E. Calculating Derivative Couplings between Time-Dependent HartreeFock Excited States with Pseudo-Wavefunctions. J. Phys. Chem. B 2015, 119 (24), 7140-9.

(40) (a) Schluns, D.; Franchini, M.; Gotz, A. W.; Neugebauer, J.; Jacob, C. R.; Visscher, L. Analytical gradients for subsystem density functional theory within the slater-function-based 3hysic3am density functional program. J. Comput. Chem. 2017, 38 (4), 238-249. (b) Sand, A. M.; Hoyer, C. E.; Sharkas, K.; Kidder, K. M.; Lindh, R.; Truhlar, D. G.; Gagliardi, L. Analytic Gradients for Complete Active Space Pair-Density Functional Theory. J. Chem. Theory Comput. 2018, 14 (1), 126-138. (c) Filatov, M.; Liu, F.; Martinez, T. J. Analytical derivatives of the individual state energies in ensemble density functional theory method. I. General formalism. J. Chem. Phys. 2017, 147 (3), 034113. (d) Ramberger, B.; Schäfer, T.; Kresse, G. Analytic interatomic forces in the random phase approximation. Phys. Rev. Lett. 2017, 118 (10), 106403.

(41) (a) Tawa, G. J.; Mielke, S. L.; Truhlar, D. G.; Schwenke, D. W. Algebraic variational and propagation formalisms for quantal dynamics calculations of electronic-to-vibrational, rotational energy transfer and application to the quenching of the $3 \mathrm{p}$ state of sodium by hydrogen molecules. J. Chem. Phys. 1994, 100 (8), 5751-5777. (b) Worth, G. A.; Meyer, H.-D.; Köppel, H.; Cederbaum, L.; Burghardt, I. Using the MCTDH wavepacket propagation method to describe multimode non-adiabatic dynamics. Int. Rev. Phys. Chem. 2008, 27 (3), 569-606. (c) Xie, Y.; Zheng, J.; Lan, Z. Fulldimensional multilayer multiconfigurational time-dependent Hartree study of electron transfer dynamics in the anthracene/C60 complex. J. Chem. Phys. 2015, 142 (8), 084706. (d) Shalashilin, D. V. Nonadiabatic dynamics with the help of multiconfigurational Ehrenfest method: Improved theory and fully quantum 24D simulation of pyrazine. J. Chem. Phys. 2010, 132 (24), 244111. (e) Wang, H. Multilayer Multiconfiguration Time-Dependent Hartree Theory. J. Phys. Chem. A 2015, 119 (29), 7951-65. (f) Worth, G. A.; Meyer, H. D.; Cederbaum, L. The effect of a model environment on the $S 2$ absorption spectrum of pyrazine: A wave packet study treating all 24 vibrational modes. J. Chem. Phys. 1996, 105 (11), 4412-4426. (g) Markmann, A.; Worth, G. A.; Mahapatra, S.; Meyer, H.-D.; Köppel, H.; Cederbaum, L. S. Simulation of a complex spectrum: Interplay of five electronic states and 21 vibrational degrees of freedom in C $5 \mathrm{H} \mathrm{4+.} \mathrm{J.} \mathrm{Chem.} \mathrm{Phys.} \mathrm{2005,} 123$ (20), 204310. (h) Worth, G.; Meyer, H.; Cederbaum, L.; Domcke, W.; Yarkony, D.; Köppel, H. Multidimensional dynamics involving a conical intersection: Wavepacket calculations using the MCTDH method. Conical intersections: Electronic structure, dynamics and spectroscopy 2004, 15, 583-617.

(42) (a) Ronca, E.; Li, Z.; Jimenez-Hoyos, C. A.; Chan, G. K. TimeStep Targeting Time-Dependent and Dynamical Density Matrix Renormalization Group Algorithms with ab Initio Hamiltonians. J. Chem. Theory Comput. 2017, 13 (11), 5560-5571. (b) Yao, Y.; Sun, K. W.; Luo, Z.; Ma, H. Full Quantum Dynamics Simulation of a Realistic Molecular System Using the Adaptive Time-Dependent Density Matrix Renormalization Group Method. J. Phys. Chem. Lett. 2018, 9 (2), 413-419. (c) Baiardi, A.; Reiher, M. Large-Scale
Quantum Dynamics with Matrix Product States. J. Chem. Theory Comput. 2019, 15 (6), 3481-3498.

(43) Richings, G. W.; Habershon, S. Direct Quantum Dynamics Using Grid-Based Wave Function Propagation and Machine-Learned Potential Energy Surfaces. J. Chem. Theory Comput. 2017, 13, 40124024.

(44) (a) Arnold, C.; Vendrell, O.; Welsch, R.; Santra, R. Control of Nuclear Dynamics through Conical Intersections and Electronic Coherences. Phys. Rev. Lett. 2018, 120 (12), 123001. (b) Liu, Y.; Martínez-Fernández, L.; Cerezo, J.; Prampolini, G.; Improta, R.; Santoro, F. Multistate coupled quantum dynamics of photoexcited cytosine in gas-phase: Nonadiabatic absorption spectrum and ultrafast internal conversions. Chem. Phys. 2018, 515, 452-463.

(45) (a) Thallmair, S.; Zauleck, J. P.; de Vivie-Riedle, R. Quantum Dynamics in an Explicit Solvent Environment: A Photochemical Bond Cleavage Treated with a Combined QD/MD Approach. J. Chem. Theory Comput. 2015, 11 (5), 1987-95. (b) Zauleck, J. P. P.; Peschel, M. T.; Rott, F.; Thallmair, S.; de Vivie-Riedle, R. Ultrafast Reactive Quantum Dynamics Coupled to Classical Solvent Dynamics Using an Ehrenfest Approach. J. Phys. Chem. A 2018, 122 (11), 2849-2857. (c) Cerezo, J.; Liu, Y.; Lin, N.; Zhao, X.; Improta, R.; Santoro, F. Mixed Quantum/Classical Method for Nonadiabatic Quantum Dynamics in Explicit Solvent Models: The pipi*/npi* Decay of Thymine in Water as a Test Case. J. Chem. Theory Comput. 2018, 14 (2), 820-832.

(46) Richings, G.; Polyak, I.; Spinlove, K.; Worth, G.; Burghardt, I.; Lasorne, B. Quantum dynamics simulations using Gaussian wavepackets: the vMCG method. Int. Rev. Phys. Chem. 2015, 34 (2), 269308.

(47) (a) Sulc, M.; Hernandez, H.; Martinez, T. J.; Vanicek, J. Relation of exact Gaussian basis methods to the dephasing representation: theory and application to time-resolved electronic spectra. J. Chem. Phys. 2013, 139 (3), 034112. (b) Hack, M. D.; Wensmann, A. M.; Truhlar, D. G.; Ben-Nun, M.; Martınez, T. J. Comparison of full multiple spawning, trajectory surface hopping, and converged quantum mechanics for electronically nonadiabatic dynamics. J. Chem. Phys. 2001, 115 (3), 1172-1186.

(48) Makhov, D. V.; Glover, W. J.; Martinez, T. J.; Shalashilin, D. V. $\mathrm{Ab}$ initio multiple cloning algorithm for quantum nonadiabatic molecular dynamics. J. Chem. Phys. 2014, 141 (5), 054110.

(49) (a) Abedi, A.; Maitra, N. T.; Gross, E. K. Exact factorization of the time-dependent electron-nuclear wave function. Phys. Rev. Lett. 2010, 105 (12), 123002. (b) Min, S. K.; Agostini, F.; Tavernelli, I.; Gross, E. K. U. Ab Initio Nonadiabatic Dynamics with Coupled Trajectories: A Rigorous Approach to Quantum (De)Coherence. J. Phys. Chem. Lett. 2017, 8 (13), 3048-3055. (c) Ha, J. K.; Lee, I. S.; Min, S. K. Surface Hopping Dynamics beyond Nonadiabatic Couplings for Quantum Coherence. J. Phys. Chem. Lett. 2018, 9 (5), 1097-1104. (d) Filatov, M.; Min, S. K.; Kim, K. S. Direct Nonadiabatic Dynamics by Mixed Quantum-Classical Formalism Connected with Ensemble Density Functional Theory Method: Application to trans-Penta-2, 4-dieniminium Cation. J. Chem. Theory Comput. 2018, 14 (9), 4499-4512.

(50) Tully, J. Mixed quantum-classical dynamics. Faraday Discuss. 1998, 110, 407-419.

(51) (a) Granucci, G.; Persico, M. Critical Appraisal of the Fewest Switches Algorithm for Surface Hopping. J. Chem. Phys. 2007, 126, 134114. (b) Subotnik, J. E.; Ouyang, W.; Landry, B. R. Can we derive Tully's surface-hopping algorithm from the semiclassical quantum Liouville equation? Almost, but only with decoherence. J. Chem. Phys. 2013, 139 (21), 214107. (c) Subotnik, J. E.; Jain, A.; Landry, B.; Petit, A.; Ouyang, W.; Bellonzi, N. Understanding the Surface Hopping View of Electronic Transitions and Decoherence. Annu. Rev. Phys. Chem. 2016, 67, 387-417. (d) Wang, L.; Akimov, A.; Prezhdo, O. V. Recent Progress in Surface Hopping: 2011-2015. J. Phys. Chem. Lett. 2016, 7, 2100. (e) Shu, Y.; Zhang, L.; Mai, S.; Sun, S.; González, L.; Truhlar, D. G. Implementation of Coherent Switching with Decay of Mixing into the SHARC program. J. Chem. Theory Comput. 2020, 16 (6), 3464-3475. (f) Zhu, C.; Jasper, A. W.; Truhlar, D. G. Non-Born- 
Oppenheimer Liouville-von Neumann dynamics. Evolution of a subsystem controlled by linear and population-driven decay of mixing with decoherent and coherent switching. J. Chem. Theory Comput. 2005, 1 (4), 527-540.

(52) (a) Meek, G. A.; Levine, B. G. Evaluation of the TimeDerivative Coupling for Accurate Electronic State Transition Probabilities from Numerical Simulations. J. Phys. Chem. Lett. 2014, 5 (13), 2351-6. (b) Plasser, F.; Granucci, G.; Pittner, J.; Barbatti, M.; Persico, M.; Lischka, H. Surface hopping dynamics using a locally diabatic formalism: charge transfer in the ethylene dimer cation and excited state dynamics in the 2-pyridone dimer. J. Chem. Phys. 2012, 137 (22), 22A514. (c) Sporkel, L.; Thiel, W. Adaptive time steps in trajectory surface hopping simulations. J. Chem. Phys. 2016, 144 (19), 194108.

(53) Menger, M.; Plasser, F.; Mennucci, B.; Gonzalez, L. Surface Hopping within an Exciton Picture. An Electrostatic Embedding Scheme. J. Chem. Theory Comput. 2018, 14 (12), 6139-6148.

(54) Mukamel, S. Principles of nonlinear optical spectroscopy; Oxford University Press on Demand: 1999.

(55) (a) Förster, T. Zwischenmolekulare energiewanderung und fluoreszenz. Ann. Phys. 1948, 437 (1-2), 55-75. (b) Förster, T. 10th Spiers Memorial Lecture. Transfer mechanisms of electronic excitation. Discuss. Faraday Soc. 1959, 27, 7-17. (c) Scholes, G. D. Long-range resonance energy transfer in molecular systems. Annu. Rev. Phys. Chem. 2003, 54 (1), 57-87.

(56) (a) Yang, M.; Fleming, G. R. Influence of phonons on exciton transfer dynamics: comparison of the Redfield, Förster, and modified Redfield equations. Chem. Phys. 2002, 282 (1), 163-180. (b) Novoderezhkin, V. I.; van Grondelle, R. Physical origins and models of energy transfer in photosynthetic light-harvesting. Phys. Chem. Chem. Phys. 2010, 12 (27), 7352-65. (c) Chenu, A.; Scholes, G. D. Coherence in energy transfer and photosynthesis. Annu. Rev. Phys. Chem. 2015, 66, 69-96. (d) Renger, T.; Muh, F. Understanding photosynthetic light-harvesting: a bottom up theoretical approach. Phys. Chem. Chem. Phys. 2013, 15 (10), 3348-71.

(57) (a) Sakamoto, S.; Tanimura, Y. Exciton-Coupled Electron Transfer Process Controlled by Non-Markovian Environments. J. Phys. Chem. Lett. 2017, 8 (21), 5390-5394. (b) Makri, N. Iterative blip-summed path integral for quantum dynamics in strongly dissipative environments. J. Chem. Phys. 2017, 146 (13), 134101. (c) Lee, M. K.; Huo, P.; Coker, D. F. Semiclassical path integral dynamics: Photosynthetic energy transfer with realistic environment interactions. Annu. Rev. Phys. Chem. 2016, 67, 639-668. (d) Strümpfer, J.; Schulten, K. Open quantum dynamics calculations with the hierarchy equations of motion on parallel computers. J. Chem. Theory Comput. 2012, 8 (8), 2808-2816. (e) Kreisbeck, C.; Kramer, T.; Aspuru-Guzik, A. Scalable high-performance algorithm for the simulation of exciton dynamics. Application to the light-harvesting complex II in the presence of resonant vibrational modes. J. Chem. Theory Comput. 2014, 10 (9), 4045-4054. (f) Kramer, T.; Noack, M.; Reinefeld, A.; Rodríguez, M.; Zelinskyy, Y. Efficient calculation of open quantum system dynamics and time-resolved spectroscopy with distributed memory HEOM (DM-HEOM). J. Comput. Chem. 2018, 39 (22), 1779-1794. (g) Richter, M.; Fingerhut, B. P. Coarse-grained representation of the quasi adiabatic propagator path integral for the treatment of non-Markovian long-time bath memory. J. Chem. Phys. 2017, 146 (21), 214101.

(58) (a) Seidner, L.; Stock, G.; Domcke, W. Nonperturbative approach to femtosecond spectroscopy: General theory and application to multidimensional nonadiabatic photoisomerization processes. J. Chem. Phys. 1995, 103 (10), 3998-4011. (b) Wolfseder, B.; Seidner, L.; Stock, G.; Domcke, W. Femtosecond pump-probe spectroscopy of electron-transfer systems: a nonperturbative approach. Chem. Phys. 1997, 217 (2-3), 275-287. (c) Gelin, M. F.; Egorova, D.; Domcke, W. Efficient method for the calculation of timeand frequency-resolved four-wave mixing signals and its application to photon-echo spectroscopy. J. Chem. Phys. 2005, 123 (16), 164112. (d) Mancal, T.; Pisliakov, A. V.; Fleming, G. R. Two-dimensional optical three-pulse photon echo spectroscopy. I. Nonperturbative approach to the calculation of spectra. J. Chem. Phys. 2006, 124 (23), 234504. (e) Ka, B. J.; Geva, E. A nonperturbative calculation of nonlinear spectroscopic signals in liquid solution. J. Chem. Phys. 2006, 125 (21), 214501.

(59) Hamm, P.; Zanni, M. Concepts and methods of 2D infrared spectroscopy; Cambridge University Press, 2011.

(60) Abramavicius, D.; Palmieri, B.; Voronine, D. V.; Sanda, F.; Mukamel, S. Coherent multidimensional optical spectroscopy of excitons in molecular aggregates; quasiparticle versus supermolecule perspectives. Chem. Rev. 2009, 109 (6), 2350-408.

(61) Koch, W.; Bonfanti, M.; Eisenbrandt, P.; Nandi, A.; Fu, B.; Bowman, J.; Tannor, D.; Burghardt, I. Two-layer Gaussian-based MCTDH study of the S1 $\leftarrow$ S0 vibronic absorption spectrum of formaldehyde using multiplicative neural network potentials. J. Chem. Phys. 2019, 151 (6), 064121.

(62) Landry, B. R.; Falk, M. J.; Subotnik, J. E. The correct interpretation of surface hopping trajectories: How to calculate electronic properties. J. Chem. Phys. 2013, 139, 211101.

(63) (a) Petit, A. S.; Subotnik, J. E. How to calculate linear absorption spectra with lifetime broadening using fewest switches surface hopping trajectories: A simple generalization of ground-state Kubo theory. J. Chem. Phys. 2014, 141 (1), 014107. (b) Petit, A. S.; Subotnik, J. E. Calculating time-resolved differential absorbance spectra for ultrafast pump-probe experiments with surface hopping trajectories. J. Chem. Phys. 2014, 141 (15), 154108. (c) Zimmermann, T.; Vanicek, J. Efficient on-the-fly ab initio semiclassical method for computing time-resolved nonadiabatic electronic spectra with surface hopping or Ehrenfest dynamics. J. Chem. Phys. 2014, 141 (13), 134102. (d) Fingerhut, B. P.; Dorfman, K. E.; Mukamel, S. Monitoring nonadiabatic dynamics of the RNA base uracil by UV pump-IR probe spectroscopy. J. Phys. Chem. Lett. 2013, 4 (11), 1933-1942. (e) Fingerhut, B. P.; Dorfman, K. E.; Mukamel, S. Probing the Conical Intersection Dynamics of the RNA Base Uracil by UV-Pump Stimulated-Raman-Probe Signals; Ab Initio Simulations. J. Chem. Theory Comput. 2014, 10 (3), 1172-1188. (f) Kowalewski, M.; Mukamel, S. Stimulated Raman signals at conical intersections: $\mathrm{Ab}$ initio surface hopping simulation protocol with direct propagation of the nuclear wave function. J. Chem. Phys. 2015, 143 (4), 044117. (g) Richter, M.; Fingerhut, B. P. Simulation of Multi-Dimensional Signals in the Optical Domain: Quantum-Classical Feedback in Nonlinear Exciton Propagation. J. Chem. Theory Comput. 2016, 12 (7), 3284-94. (h) Van Der Vegte, C.; Dijkstra, A.; Knoester, J.; Jansen, T. Calculating two-dimensional spectra with the mixed quantum-classical ehrenfest method. J. Phys. Chem. A 2013, 117 (29), 5970-5980. (i) Tempelaar, R.; van der Vegte, C. P.; Knoester, J.; Jansen, T. L. Surface hopping modeling of two-dimensional spectra. J. Chem. Phys. 2013, 138 (16), 164106.

(64) (a) Schwartz, B. J.; Rossky, P. J. Pump-probe spectroscopy of the hydrated electron: A quantum molecular dynamics simulation. $J$. Chem. Phys. 1994, 101 (8), 6917-6926. (b) Humeniuk, A.; Wohlgemuth, M.; Suzuki, T.; Mitríc, R. Time-resolved photoelectron imaging spectra from non-adiabatic molecular dynamics simulations. J. Chem. Phys. 2013, 139 (13), 134104. (c) Nenov, A.; Beccara, S.; Rivalta, I.; Cerullo, G.; Mukamel, S.; Garavelli, M. Tracking conformational dynamics of polypeptides by nonlinear electronic spectroscopy of aromatic residues: a first-principles simulation study. ChemPhysChem 2014, 15 (15), 3282-3290. (d) Giussani, A.; Marcheselli, J.; Mukamel, S.; Garavelli, M.; Nenov, A. On the Simulation of Two-dimensional Electronic Spectroscopy of Indolecontaining Peptides. Photochem. Photobiol. 2017, 93 (6), 1368-1380. (e) Nenov, A.; Giussani, A.; Fingerhut, B. P.; Rivalta, I.; Dumont, E.; Mukamel, S.; Garavelli, M. Spectral lineshapes in nonlinear electronic spectroscopy. Phys. Chem. Chem. Phys. 2015, 17 (46), 30925-30936. (f) Li, Q.; Giussani, A.; Segarra-Martí, J.; Nenov, A.; Rivalta, I.; Voityuk, A. A.; Mukamel, S.; Roca-Sanjuán, D.; Garavelli, M.; Blancafort, L. Multiple Decay Mechanisms and 2D-UV Spectroscopic Fingerprints of Singlet Excited Solvated Adenine-Uracil Monophosphate. Chem. - Eur. J. 2016, 22 (22), 7497-7507. 
(65) (a) Abramavicius, D.; Valkunas, L.; Mukamel, S. Transport and correlated fluctuations in the nonlinear optical response of excitons. Europhysics Letters (EPL) 2007, 80 (1), 17005. (b) Anda, A.; De Vico, L.; Hansen, T.; Abramavicius, D. Absorption and Fluorescence Lineshape Theory for Polynomial Potentials. J. Chem. Theory Comput. 2016, 12 (12), 5979-5989. (c) Perlik, V.; Sanda, F. Vibrational relaxation beyond the linear damping limit in two-dimensional optical spectra of molecular aggregates. J. Chem. Phys. 2017, 147 (8), 084104.

(66) Breuer, H.-P.; Petruccione, F. The theory of open quantum systems. Oxford University Press on Demand, 2002.

(67) (a) Borrego-Varillas, R.; Teles-Ferreira, D. C.; Nenov, A.; Conti, I.; Ganzer, L.; Manzoni, C.; Garavelli, M.; Maria de Paula, A.; Cerullo, G. Observation of the Sub-100 fs Population of a Dark State in a Thiobase Mediating Intersystem Crossing. J. Am. Chem. Soc. 2018, 140 (47), 16087-16093. (b) Nenov, A.; Borrego-Varillas, R.; Oriana, A.; Ganzer, L.; Segatta, F.; Conti, I.; Segarra-Marti, J.; Omachi, J.; Dapor, M.; Taioli, S.; Manzoni, C.; Mukamel, S.; Cerullo, G.; Garavelli, M. UV-Light-Induced Vibrational Coherences: The Key to Understand Kasha Rule Violation in trans-Azobenzene. J. Phys. Chem. Lett. 2018, 9 (7), 1534-1541. (c) Picchiotti, A.; Nenov, A.; Giussani, A.; Prokhorenko, V. I.; Miller, R. D.; Mukamel, S.; Garavelli, M. Pyrene, a Test Case for Deep-Ultraviolet Molecular Photophysics. J. Phys. Chem. Lett. 2019, 10 (12), 3481-3487.

(68) (a) Nenov, A.; Mukamel, S.; Garavelli, M.; Rivalta, I. TwoDimensional Electronic Spectroscopy of Benzene, Phenol, and Their Dimer: An Efficient First-Principles Simulation Protocol. J. Chem. Theory Comput. 2015, 11 (8), 3755-71. (b) Nenov, A.; Giussani, A.; Segarra-Marti, J.; Jaiswal, V. K.; Rivalta, I.; Cerullo, G.; Mukamel, S.; Garavelli, M. Modeling the high-energy electronic state manifold of adenine: Calibration for nonlinear electronic spectroscopy. J. Chem. Phys. 2015, 142 (21), 212443.

(69) (a) Fischer, S. A.; Cramer, C. J.; Govind, N. Excited State Absorption from Real-Time Time-Dependent Density Functional Theory. J. Chem. Theory Comput. 2015, 11 (9), 4294-303. (b) Nguyen, T. S.; Koh, J. H.; Lefelhocz, S.; Parkhill, J. Black-Box, Real-Time Simulations of Transient Absorption Spectroscopy. J. Phys. Chem. Lett. 2016, 7 (8), 1590-5.

(70) Plasser, F.; Ruckenbauer, M.; Mai, S.; Oppel, M.; Marquetand, P.; Gonzalez, L. Efficient and Flexible Computation of Many-Electron Wave Function Overlaps. J. Chem. Theory Comput. 2016, 12 (3), 1207-19.

(71) Delcey, M. G.; Sorensen, L. K.; Vacher, M.; Couto, R. C.; Lundberg, M. Efficient calculations of a large number of highly excited states for multiconfigurational wavefunctions. J. Comput. Chem. 2019, 40 (19), 1789-1799.

(72) (a) Teles-Ferreira, D. C.; Conti, I.; Borrego-Varillas, R.; Nenov, A.; Van Stokkum, I. H.; Ganzer, L.; Manzoni, C.; de Paula, A. M.; Cerullo, G.; Garavelli, M. A Unified Experimental/Theoretical Description of the Ultrafast Photophysics of Single and Double Thionated Uracils. Chem. - Eur. J. 2020, 26 (1), 336-343. (b) Nenov, A.; Conti, I.; Borrego-Varillas, R.; Cerullo, G.; Garavelli, M. Linear absorption spectra of solvated thiouracils resolved at the hybrid RASPT2/MM level. Chem. Phys. 2018, 515, 643-653.

(73) Mai, S.; Marquetand, P.; Gonzalez, L. Intersystem Crossing Pathways in the Noncanonical Nucleobase 2-Thiouracil: A TimeDependent Picture. J. Phys. Chem. Lett. 2016, 7 (11), 1978-83.

(74) (a) Ruckenbauer, M.; Mai, S.; Marquetand, P.; Gonzalez, L. Revealing Deactivation Pathways Hidden in Time-Resolved Photoelectron Spectra. Sci. Rep. 2016, 6, 35522. (b) Mai, S.; Marquetand, P.; Richter, M.; Gonzalez-Vazquez, J.; Gonzalez, L. Singlet and Triplet Excited-State Dynamics Study of the Keto and Enol Tautomers of Cytosine. ChemPhysChem 2013, 14, 2920-2931.

(75) Deb, S.; Weber, P. M. The ultrafast pathway of photon-induced electrocyclic ring-opening reactions: the case of 1,3-cyclohexadiene. Annu. Rev. Phys. Chem. 2011, 62, 19-39.

(76) Attar, A. R.; Bhattacherjee, A.; Pemmaraju, C. D.; Schnorr, K.; Closser, K. D.; Prendergast, D.; Leone, S. R. Femtosecond X-ray spectroscopy of an electrocyclic ring-opening reaction. Science 2017, 356 (6333), 54-59.
(77) (a) Cho, M. Coherent two-dimensional optical spectroscopy. Chem. Rev. 2008, 108 (4), 1331-418. (b) Fingerhut, B. P.; Elsaesser, T. Noncovalent Interactions of Hydrated DNA and RNA Mapped by 2D-IR Spectroscopy. In Coherent Multidimensional Spectroscopy; Cho, M.Springer, 2019; pp 171-195.

(78) (a) Egorova, D. Detection of electronic and vibrational coherences in molecular systems by $2 \mathrm{D}$ electronic photon echo spectroscopy. Chem. Phys. 2008, 347 (1-3), 166-176. (b) Butkus, V.; Zigmantas, D.; Valkunas, L.; Abramavicius, D. Vibrational vs. electronic coherences in 2D spectrum of molecular systems. Chem. Phys. Lett. 2012, 545, 40-43. (c) Butkus, V.; Valkunas, L.; Abramavicius, D. Molecular vibrations-induced quantum beats in two-dimensional electronic spectroscopy. J. Chem. Phys. 2012, 137 (4), 044513. (d) Kreisbeck, C.; Kramer, T.; Aspuru-Guzik, A. n. Disentangling electronic and vibronic coherences in two-dimensional echo spectra. J. Phys. Chem. B 2013, 117 (32), 9380-9385. (e) Egorova, D. Detection of dark states in two-dimensional electronic photon-echo signals via ground-state coherence. J. Chem. Phys. 2015, 142 (21), 212452.

(79) Segatta, F.; Cupellini, L.; Jurinovich, S.; Mukamel, S.; Dapor, M.; Taioli, S.; Garavelli, M.; Mennucci, B. A Quantum Chemical Interpretation of Two-Dimensional Electronic Spectroscopy of LightHarvesting Complexes. J. Am. Chem. Soc. 2017, 139 (22), 75587567.

(80) (a) Ostroumov, E. E.; Mulvaney, R. M.; Cogdell, R. J.; Scholes, G. D. Broadband 2D electronic spectroscopy reveals a carotenoid dark state in purple bacteria. Science 2013, 340 (6128), 52-6. (b) Ferretti, M.; Hendrikx, R.; Romero, E.; Southall, J.; Cogdell, R. J.; Novoderezhkin, V. I.; Scholes, G. D.; Van Grondelle, R. Dark states in the light-harvesting complex 2 revealed by two-dimensional electronic spectroscopy. Sci. Rep. 2016, 6, 20834.

(81) Farag, M. H.; Jansen, T. L. C.; Knoester, J. The origin of absorptive features in the two-dimensional electronic spectra of rhodopsin. Phys. Chem. Chem. Phys. 2018, 20 (18), 12746-12754.

(82) Johnson, P. J.; Farag, M. H.; Halpin, A.; Morizumi, T.; Prokhorenko, V. I.; Knoester, J.; Jansen, T. L.; Ernst, O. P.; Miller, R. $D$. The primary photochemistry of vision occurs at the molecular speed limit. J. Phys. Chem. B 2017, 121 (16), 4040-4047.

(83) Borrego-Varillas, R.; Oriana, A.; Ganzer, L.; Trifonov, A.; Buchvarov, I.; Manzoni, C.; Cerullo, G. Two-dimensional electronic spectroscopy in the ultraviolet by a birefringent delay line. Opt. Express 2016, 24 (25), 28491-28499.

(84) Kowalewski, M.; Bennett, K.; Dorfman, K. E.; Mukamel, S. Catching conical intersections in the act: Monitoring transient electronic coherences by attosecond stimulated X-ray Raman signals. Phys. Rev. Lett. 2015, 115 (19), 193003.

(85) (a) Kapteyn, H.; Cohen, O.; Christov, I.; Murnane, M. Harnessing attosecond science in the quest for coherent $\mathrm{X}$-rays. Science 2007, 317 (5839), 775-778. (b) Ullrich, J.; Rudenko, A.; Moshammer, R. Free-electron lasers: New avenues in molecular physics and photochemistry. Annu. Rev. Phys. Chem. 2012, 63, 635660. (c) Milne, C. J.; Penfold, T. J.; Chergui, M. Recent experimental and theoretical developments in time-resolved X-ray spectroscopies. Coord. Chem. Rev. 2014, 277-278, 44-68.

(86) (a) Northey, T.; Norell, J.; Fouda, A. E. A.; Besley, N. A.; Odelius, M.; Penfold, T. J. Ultrafast nonadiabatic dynamics probed by nitrogen K-edge absorption spectroscopy. Phys. Chem. Chem. Phys. 2020, 22 (5), 2667-2676. (b) Norell, J.; Eckert, S.; Van Kuiken, B. E.; Fohlisch, A.; Odelius, M. Ab initio simulations of complementary K-edges and solvatization effects for detection of proton transfer in aqueous 2-thiopyridone. J. Chem. Phys. 2019, 151 (11), 114117. (c) Segatta, F.; Nenov, A.; Orlandi, S.; Arcioni, A.; Mukamel, S.; Garavelli, M. Exploring the capabilities of optical pump X-ray probe NEXAFS spectroscopy to track photo-induced dynamics mediated by conical intersections. Faraday Discuss. 2020, 221 (0), 245-264. (d) Schweigert, I. V.; Mukamel, S. Coherent ultrafast core-hole correlation spectroscopy: $\mathrm{x}$-ray analogues of multidimensional NMR. Phys. Rev. Lett. 2007, 99 (16), 163001. (e) Hua, W.; Bennett, K.; Zhang, Y.; Luo, Y.; Mukamel, S. Study of double core hole excitations 
in molecules by X-ray double-quantum-coherence signals: a multiconfiguration simulation. Chem. Sci. 2016, 7 (9), 5922-5933. (f) Nenov, A.; Segatta, F.; Bruner, A.; Mukamel, S.; Garavelli, M. $\mathrm{X}$-ray linear and non-linear spectroscopy of the ESCA molecule. J. Chem. Phys. 2019, 151 (11), 114110. (g) Bennett, K.; Biggs, J. D.; Zhang, Y.; Dorfman, K. E.; Mukamel, S. Time-, frequency-, and wavevector-resolved $\mathrm{x}$-ray diffraction from single molecules. J. Chem. Phys. 2014, 140 (20), 204311. (h) Bennett, K.; Kowalewski, M.; Rouxel, J. R.; Mukamel, S. Monitoring molecular nonadiabatic dynamics with femtosecond X-ray diffraction. Proc. Natl. Acad. Sci. U. S. A. 2018, 115 (26), 6538-6547. (i) Ye, L.; Rouxel, J. R.; Cho, D.; Mukamel, S. Imaging electron-density fluctuations by multidimensional X-ray photon-coincidence diffraction. Proc. Natl. Acad. Sci. U. S. A. 2019, 116 (2), 395-400. (j) Parrish, R. M.; Martínez, T. J. Ab Initio Computation of Rotationally-Averaged Pump-Probe X-ray and Electron Diffraction Signals. J. Chem. Theory Comput. 2019, 15 (3), $1523-1537$.

(87) Wolf, T. J. A.; Myhre, R. H.; Cryan, J. P.; Coriani, S.; Squibb, R. J.; Battistoni, A.; Berrah, N.; Bostedt, C.; Bucksbaum, P.; Coslovich, G.; Feifel, R.; Gaffney, K. J.; Grilj, J.; Martinez, T. J.; Miyabe, S.; Moeller, S. P.; Mucke, M.; Natan, A.; Obaid, R.; Osipov, T.; Plekan, O.; Wang, S.; Koch, H.; Guhr, M. Probing ultrafast pipi*/npi* internal conversion in organic chromophores via K-edge resonant absorption. Nat. Commun. 2017, 8 (1), 29.

(88) Kukura, P.; McCamant, D. W.; Mathies, R. A. Femtosecond stimulated Raman spectroscopy. Annu. Rev. Phys. Chem. 2007, 58, 461-488.

(89) Hua, W.; Oesterling, S.; Biggs, J. D.; Zhang, Y.; Ando, H.; de Vivie-Riedle, R.; Fingerhut, B. P.; Mukamel, S. Monitoring conical intersections in the ring opening of furan by attosecond stimulated $\mathrm{X}$ ray Raman spectroscopy. Struct. Dyn. 2016, 3 (2), 023601.

(90) Zhang, Y.; Biggs, J. D.; Govind, N.; Mukamel, S. Monitoring Long-Range Electron Transfer Pathways in Proteins by Stimulated Attosecond Broadband X-ray Raman Spectroscopy. J. Phys. Chem. Lett. 2014, 5 (21), 3656-3661.

(91) (a) Zhang, Y.; Biggs, J. D.; Healion, D.; Govind, N.; Mukamel, $S$. Core and valence excitations in resonant $\mathrm{X}$-ray spectroscopy using restricted excitation window time-dependent density functional theory. J. Chem. Phys. 2012, 137 (19), 194306. (b) Lopata, K.; Van Kuiken, B. E.; Khalil, M.; Govind, N. Linear-Response and Real-Time Time-Dependent Density Functional Theory Studies of Core-Level Near-Edge X-Ray Absorption. J. Chem. Theory Comput. 2012, 8 (9), 3284-92.

(92) (a) Mata, R. A.; Suhm, M. A. Benchmarking quantum chemical methods: Are we heading in the right direction? Angew. Chem., Int. Ed. 2017, 56 (37), 11011-11018. (b) Loos, P. F.; Scemama, A.; Blondel, A.; Garniron, Y.; Caffarel, M.; Jacquemin, D. A mountaineering strategy to excited states: Highly accurate reference energies and benchmarks. J. Chem. Theory Comput. 2018, 14 (8), 4360-4379. (c) Laurent, A. D.; Jacquemin, D. TD-DFT benchmarks: a review. Int. J. Quantum Chem. 2013, 113 (17), 2019-2039. (d) Ghosh, S.; Cramer, C. J.; Truhlar, D. G.; Gagliardi, L. Generalized-active-space pair-density functional theory: an efficient method to study large, strongly correlated, conjugated systems. Chemical science 2017, 8 (4), 2741-2750. (e) Isegawa, M.; Truhlar, D. G. Valence excitation energies of alkenes, carbonyl compounds, and azabenzenes by timedependent density functional theory: Linear response of the ground state compared to collinear and noncollinear spin-flip TDDFT with the Tamm-Dancoff approximation. J. Chem. Phys. 2013, 138 (13), 134111. (f) Schreiber, M.; Silva-Junior, M. R.; Sauer, S. P.; Thiel, W. Benchmarks for electronically excited states: CASPT2, CC2, CCSD, and CC3. J. Chem. Phys. 2008, 128 (13), 134110. (g) Silva-Junior, M. R.; Schreiber, M.; Sauer, S. P.; Thiel, W. Benchmarks of electronically excited states: Basis set effects on CASPT2 results. J. Chem. Phys. 2010, 133 (17), 174318.

(93) (a) Mai, S.; Richter, M.; Ruckenbauer, M.; Oppel, M.; Marquetand, P.; Gonzalíez, L. SHARC: Surface Hopping Including Arbitrary Couplings, Program Package for Non-Adiabatic Dynamics, 2014; sharc-md.org. (b) Weingart, O.; Nenov, A.; Altoè, P.; Rivalta, I.;
Segarra-Martí, J.; Dokukina, I.; Garavelli, M. COBRAMM 2.0-A software interface for tailoring molecular electronic structure calculations and running nanoscale (QM/MM) simulations. J. Mol. Model. 2018, 24 (9), 271 site.unibo.it/cobramm/en. (c) Barbatti, M.; Ruckenbauer, M.; Plasser, F.; Pittner, J.; Granucci, G.; Persico, M.; Lischka, H. Newton-X: a surface-hopping program for nonadiabatic molecular dynamics. Wiley Interdiscip. Rev.: Comput. Mol. Sci. 2014, 4 (1), 26-33 www.univie.ac.at/newtonx.

(94) (a) Conti, I.; Garavelli, M. Evolution of the excitonic state of DNA stacked thymines: intrabase $\pi \pi^{*} \rightarrow$ S0 decay paths account for ultrafast (subpicosecond) and longer (>100 ps) deactivations. J. Phys. Chem. Lett. 2018, 9 (9), 2373-2379. (b) Giussani, A.; Conti, I.; Nenov, A.; Garavelli, M. Photoinduced formation mechanism of the thymine-thymine (6-4) adduct in DNA; a QM (CASPT2// CASSCF): MM (AMBER) study. Faraday Discuss. 2018, 207, 375387. (c) Conti, I.; Martínez-Fernández, L.; Esposito, L.; Hofinger, S.; Nenov, A.; Garavelli, M.; Improta, R. Multiple Electronic and Structural Factors Control Cyclobutane Pyrimidine Dimer and 6-4 Thymine-Thymine Photodimerization in a DNA Duplex. Chem. Eur. J. 2017, 23 (60), 15177-15188. (d) Conti, I.; Nenov, A.; Höfinger, S.; Altavilla, S. F.; Rivalta, I.; Dumont, E.; Orlandi, G.; Garavelli, M. Excited state evolution of DNA stacked adenines resolved at the CASPT2//CASSCF/Amber level: from the bright to the excimer state and back. Phys. Chem. Chem. Phys. 2015, 17 (11), 7291-7302. (e) Nenov, A.; Segarra-Martí, J.; Giussani, A.; Conti, I.; Rivalta, I.; Dumont, E.; Jaiswal, V. K.; Altavilla, S. F.; Mukamel, S.; Garavelli, M. Probing deactivation pathways of DNA nucleobases by two-dimensional electronic spectroscopy: first principles simulations. Faraday Discuss. 2015, 177, 345-362.

(95) (a) Rupp, M. Machine learning for quantum mechanics in a nutshell. Int. J. Quantum Chem. 2015, 115 (16), 1058-1073. (b) Ryczko, K.; Strubbe, D. A.; Tamblyn, I. Deep learning and density-functional theory. Phys. Rev. A: At., Mol., Opt. Phys. 2019, 100 (2), 022512. (c) Zhang, Y.-J.; Khorshidi, A.; Kastlunger, G.; Peterson, A. A. The potential for machine learning in hybrid QM/MM calculations. J. Chem. Phys. 2018, 148 (24), 241740. (d) Smith, J. S.; Nebgen, B. T.; Zubatyuk, R.; Lubbers, N.; Devereux, C.; Barros, K.; Tretiak, S.; Isayev, O.; Roitberg, A. E. Approaching coupled cluster accuracy with a general-purpose neural network potential through transfer learning. Nat. Commun. 2019, 10 (1), 2903. (e) Ahneman, D. T.; Estrada, J. G.; Lin, S.; Dreher, S. D.; Doyle, A. G. Predicting reaction performance in $\mathrm{C}-\mathrm{N}$ cross-coupling using machine learning. Science 2018, 360 (6385), 186-190. (f) Chmiela, S.; Sauceda, H. E.; Müller, K. R.; Tkatchenko, A. Towards exact molecular dynamics simulations with machine-learned force fields. Nat. Commun. 2018, 9, 3387.

(96) (a) Whitfield, J. D.; Biamonte, J.; Aspuru-Guzik, A. Simulation of electronic structure Hamiltonians using quantum computers. Mol. Phys. 2011, 109 (5), 735-750. (b) Sugisaki, K.; Nakazawa, S.; Toyota, K.; Sato, K.; Shiomi, D.; Takui, T. Quantum chemistry on quantum computers: A method for preparation of multiconfigurational wave functions on quantum computers without performing post-hartree-fock calculations. ACS Cent. Sci. 2019, 5 (1), 167-175. (c) Sugisaki, K.; Nakazawa, S.; Toyota, K.; Sato, K.; Shiomi, D.; Takui, T. Quantum chemistry on quantum computers: quantum simulations of the time evolution of wave functions under the $S 2$ operator and determination of the spin quantum number S. Phys. Chem. Chem. Phys. 2019, 21 (28), 15356-15361.

(97) McClean, J. R.; Sung, K. J.; Kivlichan, I. D.; Cao, Y.; Dai, C.; Fried, E. S.; Gidney, C.; Gimby, B.; Gokhale, P.; Häner, T. OpenFermion: the electronic structure package for quantum computers. arXiv Preprint 2017, arXiv: 1710.07629.

(98) (a) Dral, P. O.; Barbatti, M.; Thiel, W. Nonadiabatic excitedstate dynamics with machine learning. J. Phys. Chem. Lett. 2018, 9 (19), 5660-5663. (b) Chen, W.-K.; Liu, X.-Y.; Fang, W.-H.; Dral, P. O.; Cui, G. Deep Learning for Nonadiabatic Excited-State Dynamics. J. Phys. Chem. Lett. 2018, 9 (23), 6702-6708. (c) Westermayr, J.; Gastegger, M.; Menger, M. F.; Mai, S.; González, L.; Marquetand, P. Machine learning enables long time scale molecular photodynamics 
simulations. Chemical Science 2019, 10 (35), 8100-8107. (d) Yang, K. R.; Xu, X.; Truhlar, D. G. Anchor Points Reactive Potential for BondBreaking Reactions. J. Chem. Theory Comput. 2014, 10 (3), 924-933. (99) (a) Wilson, K. C.; Lyons, B.; Mehlenbacher, R.; Sabatini, R.; McCamant, D. W. Two-Dimensional Femtosecond Stimulated Raman Spectroscopy: Observation of CascadingRaman Signals in Acetonitrile. J. Chem. Phys. 2009, 131, 214502. (b) Batignani, G.; Fumero, G.; Mukamel, S.; Scopigno, T. Energy flow between spectral components in 2D broadband stimulated Raman spectroscopy. Phys. Chem. Chem. Phys. 2015, 17 (16), 10454-10461.

(100) (a) Miller, R. J. D. Mapping atomic Motions with Ultrabright Electrons: the Chemists' Gedanken Experiment Enters the Lab Frame. Annu. Rev. Phys. Chem. 2014, 65, 583-604. (b) Pande, K.; Hutchison, C. D. M.; Groenhof, G.; Aquila, A.; Robinson, J. S.; Tenboer, J.; Basu, S.; Boutet, S.; DePonte, D. P.; Liang, M.; et al. Femtosecond Structural Dynamics Drives the trans/cis Isomerization in Photoactive Yellow Protein. Science 2016, 352, 725-729. (c) Kowalewski, M.; Bennett, K.; Mukamel, S. Monitoring Nonadiabatic Avoided Crossing Dynamics in Molecules by Ultrafast X-ray Diffraction. Struct. Dyn. 2017, 4, 054101.

(101) (a) Horsch, M. T.; Chiacchiera, S.; Bami, Y.; Schmitz, G. J.; Mogni, G.; Goldbeck, G.; Ghedini, E. Reliable and interoperable computational molecular engineering: 2. Semantic interoperability based on the European Materials and Modelling Ontology. arXiv Preprint 2020, arXiv:2001.04175. (b) Horsch, M. T.; Chiacchiera, S.; Seaton, M. A.; Todorov, I. T.; Schembera, B.; Konchakova, N.; Klein, $\mathrm{P}$., Reliable and interoperable computational molecular engineering: 1. Pragmatic interoperability and translation of industrial engineering problems into modelling and simulation solutions. Molecular and Mesoscopic Modelling in Chemical Engineering Data Science, 2020.

(102) Arute, F.; Arya, K.; Babbush, R.; Bacon, D.; Bardin, J. C.; Barends, R.; Biswas, R.; Boixo, S.; Brandao, F. G. S. L.; Buell, D. A.; Burkett, B.; Chen, Y.; Chen, Z.; Chiaro, B.; Collins, R.; Courtney, W.; Dunsworth, A.; Farhi, E.; Foxen, B.; Fowler, A.; Gidney, C.; Giustina, M.; Graff, R.; Guerin, K.; Habegger, S.; Harrigan, M. P.; Hartmann, M. J.; Ho, A.; Hoffmann, M.; Huang, T.; Humble, T. S.; Isakov, S. V.; Jeffrey, E.; Jiang, Z.; Kafri, D.; Kechedzhi, K.; Kelly, J.; Klimov, P. V.; Knysh, S.; Korotkov, A.; Kostritsa, F.; Landhuis, D.; Lindmark, M.; Lucero, E.; Lyakh, D.; Mandrà, S.; McClean, J. R.; McEwen, M.; Megrant, A.; Mi, X.; Michielsen, K.; Mohseni, M.; Mutus, J.; Naaman, O.; Neeley, M.; Neill, C.; Niu, M. Y.; Ostby, E.; Petukhov, A.; Platt, J. C.; Quintana, C.; Rieffel, E. G.; Roushan, P.; Rubin, N. C.; Sank, D.; Satzinger, K. J.; Smelyanskiy, V.; Sung, K. J.; Trevithick, M. D.; Vainsencher, A.; Villalonga, B.; White, T.; Yao, Z. J.; Yeh, P.; Zalcman, A.; Neven, H.; Martinis, J. M. Quantum supremacy using a programmable superconducting processor. Nature 2019, 574 (7779), $505-510$. 